\title{
On Efficiency and Localisation for the Torsion Function
}

\author{
M. van den Berg ${ }^{1} \cdot$ D. Bucur ${ }^{2} \cdot$ T. Kappeler ${ }^{3}$
}

Received: 3 July 2020 / Accepted: 26 April 2021 / Published online: 19 May 2021

(C) The Author(s) 2021

\begin{abstract}
We consider the torsion function for the Dirichlet Laplacian $-\Delta$, and for the Schrödinger operator $-\Delta+V$ on an open set $\Omega \subset \mathbb{R}^{m}$ of finite Lebesgue measure $0<|\Omega|<\infty$ with a real-valued, non-negative, measurable potential $V$. We investigate the efficiency and the phenomenon of localisation for the torsion function, and their interplay with the geometry of the first Dirichlet eigenfunction.
\end{abstract}

Keywords Torsion function · First Dirichlet eigenfunction · Schrödinger operator ·

Dirichlet boundary condition · Localisation · Efficiency

Mathematics Subject Classification (2010) 35J05 · 35J25 $\cdot 35 \mathrm{~K} 05 \cdot 35 \mathrm{P} 99$

\section{Introduction and Main Results}

Let $\Omega$ be an open set in $\mathbb{R}^{m}$, with finite Lebesgue measure, $0<|\Omega|<\infty$, and boundary $\partial \Omega$, and let

$$
L=-\Delta+V
$$

be the Schrödinger operator acting in $L^{2}(\Omega)$ with the potential $V: \Omega \rightarrow \mathbb{R}^{+}, \mathbb{R}^{+}=[0, \infty)$ being measurable. The torsion function for $\Omega$ is the unique solution of

$$
-\Delta v=1, \quad v \in H_{0}^{1}(\Omega) .
$$

M. van den Berg

mamvdb@bristol.ac.uk

D. Bucur

dorin.bucur@univ-savoie.fr

T. Kappeler

thomas.kappeler@math.uzh.ch

1 School of Mathematics, University of Bristol, Fry Building, Woodland Road, Bristol, BS8 1UG, UK

2 UMR CNRS 5127, Campus Scientifique, Laboratoire de Mathématiques, Université Savoie Mont Blanc, 73376 Le-Bourget-Du-Lac, France

3 Institut für Mathematik, Universität Zürich, Winterthurerstrasse 190, CH-8057 Zürich, Switzerland 
It is denoted by $v_{\Omega}$, and is also referred to as the torsion function for the Dirichlet Laplacian. The function $v_{\Omega}$ is non-negative, pointwise increasing in $\Omega$, and satisfies,

$$
\lambda_{1}(\Omega)^{-1}<\left\|v_{\Omega}\right\|_{\infty} \leq(4+3 m \log 2) \lambda_{1}(\Omega)^{-1},
$$

where

$$
\lambda_{1}(\Omega)=\inf _{\varphi \in H_{0}^{1}(\Omega) \backslash\{0\}} \frac{\|\nabla \varphi\|_{2}^{2}}{\|\varphi\|_{2}^{2}},
$$

is the first eigenvalue of the Dirichlet Laplacian. Here, and throughout the paper, $\|\cdot\|_{p}$ denotes the standard $L^{p}$ norm, $1 \leq p \leq \infty$. Since $|\Omega|<\infty$ the first eigenvalue is bounded away from 0 by the Faber-Krahn inequality. The $m$-dependent constant in the right-hand side of Eq. 1 has subsequently been improved [17, 24]. We denote the sharp constant by

$$
\mathfrak{c}_{m}=\sup \left\{\lambda_{1}(\Omega)\left\|v_{\Omega}\right\|_{\infty}: \Omega \text { open in } \mathbb{R}^{m}, 0<|\Omega|<\infty\right\} .
$$

More generally, the equation $L v=1$ also has a unique solution $v_{\Omega, V} \in H_{0}^{1}(\Omega)$, referred to as the torsion function for $L$.

In this paper we study the efficiency of the torsion function of Schrödinger operators, and study the phenomenon of localisation. The notion of efficiency, or mean to max ratio, goes back to [21, 23], where it was introduced for the first Dirichlet eigenfunction. It can be viewed as a (rough) measure of localisation. The mean to max ratio for the torsion function for bounded, open, convex sets in Euclidean space was studied in [15] in the more general context of the $p$-torsional rigidity. The phenomenon of localisation of eigenfunctions of Schrödinger operators is a prominent and very active research area and has important applications in the applied sciences. The literature is extensive. See for example the review paper [18]. It was discovered in [1, 2], and the references therein, that under appropriate conditions, $v_{\Omega, V}^{-1}$ can be used for approximating eigenvalues and eigenfunctions of $L$. It raises the question as to whether under appropriate assumptions, the phenomenon of localisation can also be observed for the torsion function of Schrödinger operators, and suggests to investigate the interplay between the localisation of the torsion function and the one of the first Dirichlet eigenfunction.

The main results of this papers can be described in an informal way as follows. Theorem 1 compares the efficiency of $v_{\Omega, V}$ with the one of $v_{\Omega}$ under a variety of hypotheses. In addition it shows that for any given $\Omega$, the efficiency for $v_{\Omega, V}$ can be arbitrarily close to 1 . Theorem 2 asserts that the efficiency for the first eigenfunction of the Dirichlet Laplacian can be arbitrarily close to 1 . Among other results, Theorem 3 provides a quantitative estimate, showing that in case the efficiency for the first eigenfunction of the Dirichlet Laplacian is close to one, the corresponding first eigenvalue is large. Finally, Theorem 4 shows that localisation for the torsion function of the Dirichlet Laplacian implies localisation for the first eigenfunction of this operator.

Definition 1 (i) Let $\Omega$ be an open set in $\mathbb{R}^{m}$ with $0<|\Omega|<\infty$, and let $f: \Omega \rightarrow[0, \infty)$, with $0<\|f\|_{\infty}<\infty$. The mean to max ratio of $f$ is the real number $\frac{\|f\|_{1}}{|\Omega|\|f\|_{\infty}}$.

(ii) If $v_{\Omega, V}$ is the torsion function for $L$, then its efficiency is its mean to max ratio,

$$
\Phi(\Omega, V)=\frac{\left\|v_{\Omega, V}\right\|_{1}}{|\Omega|\left\|v_{\Omega, V}\right\|_{\infty}} .
$$

If $V=0$, then $\Phi(\Omega, 0)$ is denoted by $\Phi(\Omega)$, which coincides with the definition in [15]. 
Our first result concerns the comparison of $\Phi(\Omega, V)$ and $\Phi(\Omega)$. In [19] it was shown that if $m \geq 2$, then

$$
\inf \left\{\Phi(\Omega): \Omega \subset \mathbb{R}^{m}, \Omega \text { open, } 0<|\Omega|<\infty\right\}=0,
$$

and

$$
\sup \left\{\Phi(\Omega): \Omega \subset \mathbb{R}^{m}, \Omega \text { open, } 0<|\Omega|<\infty\right\}=1 .
$$

The analogous result for Schrödinger operators is stated under (ii) of the theorem below.

Theorem 1 (i) If $\Omega$ is an open set in $\mathbb{R}^{m}$ with $0<|\Omega|<\infty$, and if $V: \Omega \rightarrow \mathbb{R}^{+}$is measurable with $0 \leq V \leq c, c>0$, then

$$
\Phi(\Omega, V) \leq 2^{2(3 m+4) c / \lambda_{1}(\Omega)} \frac{8 c+\lambda_{1}(\Omega)}{\lambda_{1}(\Omega)}\left(\frac{8 c+\lambda_{1}(\Omega)}{8 c}\right)^{8 c / \lambda_{1}(\Omega)} \Phi(\Omega),
$$

and

$$
\Phi(\Omega, V) \geq 2^{-2(3 m+4) c / \lambda_{1}(\Omega)} \frac{\lambda_{1}(\Omega)}{8 c+\lambda_{1}(\Omega)}\left(\frac{8 c}{8 c+\lambda_{1}(\Omega)}\right)^{8 c / \lambda_{1}(\Omega)} \Phi(\Omega) .
$$

Furthermore for fixed $\Omega$ the right-hand sides of Eqs. 4 and 5 converge to $\Phi(\Omega)$ as $c \downarrow 0$.

(ii) Let $\left(\Omega_{n}\right)$ be a sequence of open sets in $\mathbb{R}^{m}$ with $0<\left|\Omega_{n}\right|<\infty$, and let $V_{n}$ : $\Omega_{n} \rightarrow \mathbb{R}^{+}$be a sequence of measurable functions. If there exists $\eta<\infty$ such that $\sup _{n \in \mathbb{N}} \frac{\left\|V_{n}\right\|_{\infty}}{\lambda_{1}\left(\Omega_{n}\right)} \leq \eta$, then $\lim _{n \rightarrow \infty} \Phi\left(\Omega_{n}, V_{n}\right)=0$ if and only if $\lim _{n \rightarrow \infty} \Phi\left(\Omega_{n}\right)=0$.

(iii) If $\Omega$ is a fixed open set in $\mathbb{R}^{m}$ with $0<|\Omega|<\infty$, then

$$
\sup \left\{\Phi(\Omega, V):\left(V: \Omega \rightarrow \mathbb{R}^{+}, \text {measurable }\right)\right\}=1,
$$

and

$$
\inf \left\{\Phi(\Omega, V):\left(V: \Omega \rightarrow \mathbb{R}^{+}, \text {measurable }\right)\right\}=\Phi(\Omega)
$$

In fact,

$$
1>\Phi(\Omega, c) \geq 1-\frac{2^{(m+4) / 2}}{|\Omega|} \int_{\Omega} d x e^{-c^{1 / 2} d_{\Omega}(x) / 2}, \quad \forall c \geq 0,
$$

where $d_{\Omega}: \Omega \rightarrow \mathbb{R}^{+}$is the distance to the boundary function,

$$
d_{\Omega}(x)=\min \left\{|x-y|: y \in \mathbb{R}^{m} \backslash \Omega\right\} .
$$

We denote the spectrum of $L$ with Dirichlet boundary conditions by

$$
\left\{\lambda_{1}(\Omega, V) \leq \lambda_{2}(\Omega, V) \leq \cdots\right\},
$$

accumulating at infinity only, and choose a corresponding $L^{2}$-orthonormal basis of eigenfunctions $\left\{\varphi_{1, \Omega, V}, \varphi_{2, \Omega, V}, \cdots\right\}$. If the first Dirichlet eigenvalue $\lambda_{1}(\Omega, V)$ of $L$ has multiplicity 1 , then its corresponding eigenspace is one-dimensional, and $\varphi_{1, \Omega, V}$ is uniquely defined up to a sign. Since $\varphi_{1, \Omega, V}$ does not change sign we may choose $\varphi_{1, \Omega, V}>0$. In that case we denote the efficiency, or mean to max ratio, of $\varphi_{1, \Omega, V}$ by

$$
E(\Omega, V)=\frac{\left\|\varphi_{1, \Omega, V}\right\|_{1}}{|\Omega|\left\|\varphi_{1, \Omega, V}\right\|_{\infty}} .
$$

If $V=0$, then $E(\Omega, 0)$ is denoted by $E(\Omega)$, which coincides with the definition on p.92 in [23]. See also [7]. We note that if $\Omega$ is connected, then $\lambda_{1}(\Omega)$ is simple. 
By [8, Theorem 1.2] and [5, Theorem 1] it is possible for $m \geq 2$ to construct, for any $\varepsilon \in(0,1)$, an open connected set $\Omega_{\varepsilon} \subset \mathbb{R}^{m}$ with $0<\left|\Omega_{\varepsilon}\right|<\infty$ such that both

$$
\frac{\lambda_{1}\left(\Omega_{\varepsilon}\right)\left\|v_{\Omega_{\varepsilon}}\right\|_{1}}{\left|\Omega_{\varepsilon}\right|}>1-\varepsilon,
$$

and

$$
\lambda_{1}\left(\Omega_{\varepsilon}\right)\left\|v_{\Omega_{\varepsilon}}\right\|_{\infty}<1+\varepsilon
$$

This implies that

$$
\Phi\left(\Omega_{\varepsilon}\right) \geq \frac{1-\varepsilon}{1+\varepsilon}, \quad \forall \varepsilon \in(0,1),
$$

which in turn implies (3). Given $\varepsilon \in(0,1)$, we were unable to construct a set $\Omega_{\varepsilon}$ such that $E\left(\Omega_{\varepsilon}\right)>1-\varepsilon$. Nevertheless we have the following:

Theorem 2 If $m \geq 2$, then

$$
\sup \left\{E(\Omega): \Omega \subset \mathbb{R}^{m}, \Omega \text { open and connected, } 0<|\Omega|<\infty\right\}=1 .
$$

By examining the proof of Theorem 2 in Section 4 we see that for any $m \geq 2$, and $\varepsilon \in(0,1)$ there exists an open, bounded and connected set $\Omega_{\varepsilon} \subset \mathbb{R}^{m}$ such that (i) $E\left(\Omega_{\varepsilon}\right) \geq$ $1-\varepsilon$, and (ii) $\lambda_{1}\left(\Omega_{\varepsilon}\right)\left|\Omega_{\varepsilon}\right|^{2 / m}$ is large for $\varepsilon$ small. In Theorem 3 below we show that this is a general phenomenon. That is if $\Omega$ is any open and connected set in $\mathbb{R}^{m}, m \geq 2$, with $0<|\Omega|<\infty$ such $E(\Omega)$ is close to 1 , then the eigenfunction is close to its maximum on most of $\Omega$, and $\lambda_{1}(\Omega)|\Omega|^{2 / m}$ is large. We have a similar phenomenon for the torsion function. Throughout we denote by $B(p ; R)=\left\{x \in \mathbb{R}^{m}:|x-p|<R\right\}$ the open ball with centre $p$ and radius $R$. We put $B_{R}=B(0 ; R)$, and $\omega_{m}=\left|B_{1}\right|$. For $\Omega$ open with $0<|\Omega|<\infty$, and $u \in L^{1}(\Omega)$,

$$
f_{\Omega} u:=\frac{1}{|\Omega|} \int_{\Omega} u
$$

Theorem 3 Let $m \geq 2$ and let $\Omega$ be a non-empty open set in $\mathbb{R}^{m}$ with finite Lebesgue measure, $|\Omega|<\infty$.

(i) If $u \in H_{0}^{1}(\Omega) \cap L^{\infty}(\Omega),\|u\|_{\infty}>0$, and if

$$
f_{\Omega} u \geq \frac{2\|u\|_{\infty}}{m+2}
$$

then

$$
\left(\frac{\omega_{m}}{|\Omega|}\right)^{(2-m) / 2}\left(\|u\|_{\infty}-f_{\Omega} u\right) \int_{\Omega}|\nabla u|^{2} \geq \frac{4 m^{2}}{(m+2)^{2}} \omega_{m}\|u\|_{\infty}^{3} .
$$

Equality occurs if and only if $\Omega$ is a ball, and $u$ is a multiple of the torsion function. (ii)

$$
\begin{aligned}
& \left\|v_{\Omega}\right\|_{\infty} \leq \frac{(m+2)^{2}}{4 m^{2}}\left(\frac{|\Omega|}{\omega_{m}}\right)^{2 / m}(1-\Phi(\Omega)), \\
& \lambda_{1}(\Omega) \geq \frac{4 m^{2}}{(m+2)^{2}}\left(\frac{\omega_{m}}{|\Omega|}\right)^{2 / m}(1-\Phi(\Omega))^{-1} .
\end{aligned}
$$


(iii) If $\Omega$ is connected, then

$$
\lambda_{1}(\Omega) \geq \frac{4 m^{2}}{(m+2)^{2}}\left(\frac{\omega_{m}}{|\Omega|}\right)^{2 / m}(1-E(\Omega))^{-1} .
$$

The last result of this paper concerns the localisation of a sequence of torsion functions. We make the following definition.

Definition 2 Let $\left(\Omega_{n}\right)$ be a sequence of open sets in $\mathbb{R}^{m}$ with $0<\left|\Omega_{n}\right|<\infty$, and let

$$
\mathfrak{A}\left(\left(\Omega_{n}\right)\right)=\left\{\left(A_{n}\right):(\forall n \in \mathbb{N})\left(A_{n} \subset \Omega_{n}, A_{n} \text { measurable }\right), \lim _{n \rightarrow \infty} \frac{\left|A_{n}\right|}{\left|\Omega_{n}\right|}=0\right\} .
$$

Let $1 \leq p<\infty$. For $n \in \mathbb{N}, f_{n} \in L^{p}\left(\Omega_{n}\right), f_{n} \geq 0$, and $f_{n} \neq 0$, we define

$$
\kappa=\sup \left\{\limsup _{n \rightarrow \infty} \frac{\left\|f_{n} \mathbf{1}_{A_{n}}\right\|_{p}^{p}}{\left\|f_{n}\right\|_{p}^{p}}:\left(A_{n}\right) \in \mathfrak{A}\left(\left(\Omega_{n}\right)\right)\right\} .
$$

(i) We say that the sequence $\left(f_{n}\right) \kappa$-localises in $L^{p}$ if $0<\kappa<1$.

(ii) We say that the sequence $\left(f_{n}\right)$ localises in $L^{p}$ if $\kappa=1$.

(iii) We say that the sequence $\left(f_{n}\right)$ does not localise in $L^{p}$ if $\kappa=0$.

Using Cantor's diagonalisation we see the supremum in Eq. 13 is in fact a maximum. That is we have a maximising sequence in $\mathfrak{A}\left(\left(\Omega_{n}\right)\right)$. To show that $\left(f_{n}\right)$ localises in $L^{p}$ is equivalent to showing the existence of a sequence of measurable sets $A_{n} \subset \Omega_{n}, n \in \mathbb{N}$ such that

$$
\lim _{n \rightarrow \infty} \frac{\left|A_{n}\right|}{\left|\Omega_{n}\right|}=0, \quad \lim _{n \rightarrow \infty} \frac{\left\|f_{n} \mathbf{1}_{A_{n}}\right\|_{p}^{p}}{\left\|f_{n}\right\|_{p}^{p}}=1 .
$$

To show that $\left(f_{n}\right)$ does not localise in $L^{p}$ is equivalent to showing that for any sequence ( $A_{n}$ ) of measurable sets $A_{n} \subset \Omega_{n}, n \in \mathbb{N}$ the following implication holds:

$$
\lim _{n \rightarrow \infty} \frac{\left|A_{n}\right|}{\left|\Omega_{n}\right|}=0 \Rightarrow \lim _{n \rightarrow \infty} \frac{\left\|f_{n} \mathbf{1}_{A_{n}}\right\|_{p}}{\left\|f_{n}\right\|_{p}}=0 .
$$

If $\left(f_{n}\right) \kappa$-localises in $L^{p}$ then there is a sequence $\left(A_{n}\right) \in \mathfrak{A}\left(\left(\Omega_{n}\right)\right)$ which (asymptotically) supports a fraction $\kappa$ of $\left\|f_{n}\right\|_{p}^{p}$. Given such a maximising sequence $\left(A_{n}\right)$ it is possible to construct a sequence $\left(\tilde{A_{n}}\right) \in \mathfrak{A}\left(\left(\Omega_{n}\right)\right)$ which (asymptotically) supports a fraction $\tilde{\kappa}$ of $\left\|f_{n}\right\|_{p}^{p}$ with $0<\tilde{\kappa}<\kappa$. Hence the requirement of the supremum in the definition of $\kappa$ in Eq. 13. In Section 2 we analyse two examples in detail where localisation in $L^{1}$ and $\kappa$-localisation in $L^{1}$ occur for a family of torsion functions for Schrödinger operators (Example 1), and for a family of torsion functions for Dirichlet Laplacians (Example 2).

The theorem below asserts that localisation or $\kappa$-localisation for the torsion function in $L^{1}$ implies localisation for the corresponding first Dirichlet eigenfunction in $L^{2}$.

Theorem 4 Let $\left(\Omega_{n}\right)$ be a sequence of open sets in $\mathbb{R}^{m}$ with $0<\left|\Omega_{n}\right|<\infty$ and let $V_{n}: \Omega_{n} \rightarrow \mathbb{R}^{+}$be a sequence of measurable functions. If $\left(v_{\Omega_{n}, V_{n}}\right)$ either localises or $\kappa-$

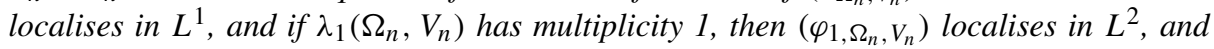
$\lim _{n \rightarrow \infty} \Phi\left(\Omega_{n}, V_{n}\right)=\lim _{n \rightarrow \infty} E\left(\Omega_{n}, V_{n}\right)=0$. 
It follows from Theorem 1.1 in [15] that, when restricting $\Omega$ to be open, bounded and convex in $\mathbb{R}^{m}, m \geq 1$, one has

$$
\frac{2}{m(m+2)} \leq \inf \left\{\Phi(\Omega): \Omega \subset \mathbb{R}^{m}, \Omega \text { open, convex, } 0<|\Omega|<\infty\right\} .
$$

We see by Eq. 16 that for any sequence $\left(\Omega_{n}\right)$ of elongating open, bounded and convex sets in $\mathbb{R}^{m},\left(v_{\Omega_{n}}\right)$ has non-vanishing efficiency, and so by Theorem $4,\left(v_{\Omega_{n}}\right)$ is not localising or $\kappa$-localising in $L^{1}$. This is in contrast with the results of [7], where localisation of a sequence of first Dirichlet eigenfunctions was obtained for a wide class of elongating, open, bounded and convex sets in $\mathbb{R}^{m}$. Further examples demonstrating different behaviour of the torsion function, and the first eigenfunction of the Dirichlet Laplacian around their respective maxima for elongated convex planar domains have been constructed in [3].

This paper is organised as follows. Examples 1 and 2 will be analysed in Section 2. The proofs of Theorems 1, 2, 3 and 4 will be given in Sections 3-6 respectively.

\section{Examples}

In Example 1 below we analyse localisation and $\kappa$-localisation in $L^{1}$ for a family of Schrödinger operators in one dimension, parametrised by three real numbers, $v>1$, $0<\alpha<1$, and $c>0$.

Example 1 Let $\Omega=(-1,1), v>1$, and $0<\varepsilon<1$. Denote by $V(x) \equiv V_{\nu, \varepsilon}(x)$ the potential

$$
V(x)=v^{2} \mathbf{1}_{(-1,-\varepsilon)}(x)+v^{2} \mathbf{1}_{(\varepsilon, 1)}(x), \quad x \in(-1,1),
$$

and by $v_{v, \varepsilon}(x) \equiv v_{(-1,1), V_{v, \varepsilon}}(x)$ the torsion function for $-\Delta+V$ on $(-1,1)$ with Dirichlet boundary conditions. If $\varepsilon_{\alpha}(v)=\frac{c}{v^{\alpha}}$ with $0<\alpha<1, c>0$, and $v>1$ sufficiently large so that $0<\varepsilon_{\alpha}(v)<1$, then the following holds:

(i) If $\frac{2}{3}<\alpha<1$, then $\left(v_{n, \varepsilon_{\alpha}(n)}\right)$ does not localise in $L^{1}, \kappa=0$.

(ii) If $0<\alpha<\frac{2}{3}$, then $\left(v_{n, \varepsilon_{\alpha}(n)}\right)$ localises in $L^{1}, \kappa=1$.

(iii) If $\alpha=\frac{2}{3}$, then $\left(v_{n, \varepsilon_{\alpha}(n)}\right) \kappa_{c}$-localises in $L^{1}$ with

$$
\kappa_{c}=\frac{c^{3} / 3}{1+c^{3} / 3} .
$$

Proof Since the potential $V$ is even, so is the torsion function $v \equiv v_{v, \varepsilon}$ and hence it suffices to determine $v$ on $[0,1]$. On the interval $[0, \varepsilon]$, the function $v$ is of the form

$$
v_{1}(x):=-\frac{1}{2} x^{2}+\gamma, \quad 0 \leq x \leq \varepsilon, \quad \gamma \equiv \gamma_{\nu, \varepsilon},
$$

whereas on the interval $[\varepsilon, 1]$, we make the following Ansatz:

$$
v_{2}(x):=\frac{1}{v^{2}}-\alpha e^{v x}+\beta e^{-v x}, \quad \varepsilon \leq x \leq 1, \quad \alpha \equiv \alpha_{v, \varepsilon}, \beta \equiv \beta_{v, \varepsilon} .
$$

It is straightforward to verify that

$$
(-\Delta+V) v_{1}=-\Delta v_{1}=1, \quad 0 \leq x \leq \varepsilon,
$$

and

$$
(-\Delta+V) v_{2}=-v^{2}\left(v_{2}-\frac{1}{v^{2}}\right)+v^{2} v_{2}=1, \quad \varepsilon \leq x \leq 1 .
$$


The constants $\gamma, \alpha$, and $\beta$ are determined by the boundary condition $v_{2}(1)=0$, and the matching conditions

$$
v_{1}(\varepsilon)=v_{2}(\varepsilon), \quad v_{1}^{\prime}(\varepsilon)=v_{2}^{\prime}(\varepsilon),
$$

where ' denotes the derivative with respect to the variable $x$. The boundary condition $v_{2}(1)=0$ yields $\frac{1}{v^{2}}-\alpha e^{v}+\beta e^{-v}=0$ or

$$
\beta=\alpha e^{2 v}-\frac{1}{v^{2}} e^{v} .
$$

The matching condition $v_{1}^{\prime}(\varepsilon)=v_{2}^{\prime}(\varepsilon)$ reads $\varepsilon=v \alpha e^{v \varepsilon}+v \beta e^{-v \varepsilon}$ or, with Eq. 18,

$$
\alpha=\frac{\varepsilon+\frac{1}{\nu} e^{\nu(1-\varepsilon)}}{v\left(e^{\nu \varepsilon}+e^{2 \nu-\nu \varepsilon}\right)}=\frac{1}{v^{2} e^{\nu}} \frac{1+v \varepsilon e^{-v(1-\varepsilon)}}{1+e^{-2 v(1-\varepsilon)}} .
$$

The leading term of $\alpha$ is thus $\frac{1}{v^{2} e^{v}}$,

$$
\alpha=\frac{1}{v^{2} e^{\nu}}+\frac{\varepsilon}{v e^{\nu(2-\varepsilon)}} \frac{1-\frac{1}{\nu \varepsilon} e^{-v(1-\varepsilon)}}{1+e^{-2 \nu(1-\varepsilon)}} .
$$

Combining (18) and (20) we obtain the following formula for $\beta$,

$$
\beta=\frac{\varepsilon e^{\nu \varepsilon}}{v} \frac{1-\frac{1}{\nu \varepsilon} e^{-v(1-\varepsilon)}}{1+e^{-2 v(1-\varepsilon)}}=\frac{\varepsilon e^{\nu \varepsilon}}{v}-\frac{e^{\nu \varepsilon}}{v^{2}} \frac{1}{e^{\nu(1-\varepsilon)}} \frac{1+\nu \varepsilon e^{-v(1-\varepsilon)}}{1+e^{-2 v(1-\varepsilon)}} .
$$

Finally the matching condition $v_{1}(\varepsilon)=v_{2}(\varepsilon)$ reads as

$$
-\frac{1}{2} \varepsilon^{2}+\gamma=\frac{1}{v^{2}}-\alpha e^{\nu \varepsilon}+\beta e^{-v \varepsilon},
$$

which, when combined with Eqs. 19 and 21, yields the following formula for $\gamma$

$$
\gamma=\frac{1}{2} \varepsilon^{2}+\frac{\varepsilon}{v}+\frac{1}{v^{2}}-\frac{2}{v^{2} e^{\nu(1-\varepsilon)}} \frac{1+v \varepsilon e^{-v(1-\varepsilon)}}{1+e^{-2 v(1-\varepsilon)}} .
$$

Now let us compute

$$
\frac{\int_{\varepsilon}^{1} v}{\int_{0}^{1} v}=\frac{\int_{\varepsilon}^{1} v_{2}}{\int_{0}^{\varepsilon} v_{1}+\int_{\varepsilon}^{1} v_{2}}
$$

We have $\int_{0}^{\varepsilon} v_{1}=\left.\left(-\frac{1}{6} x^{3}+\gamma x\right)\right|_{0} ^{\varepsilon}$, yielding

$$
\int_{0}^{\varepsilon} v_{1}=\frac{\varepsilon^{3}}{3}+\frac{\varepsilon^{2}}{v}+\frac{\varepsilon}{v^{2}}-\frac{2 \varepsilon}{v^{2} e^{\nu(1-\varepsilon)}} \frac{1+v \varepsilon e^{-v(1-\varepsilon)}}{1+e^{-2 v(1-\varepsilon)}} .
$$

Similarly, we compute

$$
\int_{\varepsilon}^{1} v_{2}=\frac{1-\varepsilon}{v^{2}}-\frac{\alpha}{v}\left(e^{v}-e^{\nu \varepsilon}\right)-\frac{\beta}{v}\left(e^{-v}-e^{-v \varepsilon}\right),
$$

which yields

$$
\int_{\varepsilon}^{1} v_{2}=\frac{1}{v^{2}}-\frac{1}{v^{3}}-\frac{2 \varepsilon}{v^{2} e^{v(1-\varepsilon)}} \frac{1-\frac{1}{\nu \varepsilon} e^{-v(1-\varepsilon)}}{1+e^{-2 v(1-\varepsilon)}} .
$$

Combining (23) and (24), one obtains in the case where $\varepsilon=\frac{c}{v^{\alpha}}$,

$$
\int_{0}^{\varepsilon} v_{1}+\int_{\varepsilon}^{1} v_{2}=\frac{c^{3}}{3 v^{3 \alpha}}+\frac{1}{v^{2}}+O\left(v^{-1-2 \alpha}\right), v \rightarrow \infty,
$$


and hence

$$
\lim _{\nu \rightarrow \infty} \frac{\int_{\varepsilon}^{1} v_{2}}{\int_{0}^{\varepsilon} v_{1}+\int_{\varepsilon}^{1} v_{2}}= \begin{cases}1 & \text { if } \frac{2}{3}<\alpha<1, \\ 0 & \text { if } 0<\alpha<\frac{2}{3}, \\ \frac{1}{1+c^{3} / 3} & \text { if } \alpha=\frac{2}{3} .\end{cases}
$$

This implies that

$$
\lim _{\nu \rightarrow \infty} \frac{\int_{-\varepsilon}^{\varepsilon} v}{\int_{-1}^{1} v}=1-\lim _{\nu \rightarrow \infty} \frac{\int_{\varepsilon}^{1} v_{2}}{\int_{0}^{\varepsilon} v_{1}+\int_{\varepsilon}^{1} v_{2}}= \begin{cases}0 & \text { if } \frac{2}{3}<\alpha<1, \\ 1 & \text { if } 0<\alpha<\frac{2}{3}, \\ \frac{c^{3} / 3}{1+c^{3} / 3} & \text { if } \alpha=\frac{2}{3} .\end{cases}
$$

To prove (i) we let $A_{n} \subset(-1,1), n \in \mathbb{N}$ be an arbitrary sequence of measurable sets which satisfy $\lim _{n \rightarrow \infty}\left|A_{n}\right|=0$. Since $v_{n} \equiv v_{n, \varepsilon_{\alpha}(n)}$ is even, it follows that, with $\varepsilon_{n} \equiv \varepsilon_{\alpha}(n)$,

$$
\int_{A_{n}} v_{n} \leq 2 \int_{0}^{\varepsilon_{n}} v_{n}+2 \int_{\varepsilon_{n}}^{2 \varepsilon_{n}} v_{n}+\int_{A_{n} \cap\left[2 \varepsilon_{n}, 1\right]} v_{n}+\int_{A_{n} \cap\left[-1,-2 \varepsilon_{n}\right]} v_{n} .
$$

First note that by Eq. 25

$$
\int_{0}^{\varepsilon_{n}} v_{n}+\int_{\varepsilon_{n}}^{1} v_{n}=\frac{1}{n^{2}}+O\left(n^{-3 \alpha}\right), v \rightarrow \infty .
$$

By Eq. $26 \lim _{n \rightarrow \infty} \frac{\int_{0}^{\varepsilon n} v_{n}}{\int_{0}^{1} v_{n}}=0$. Since for $n$ sufficiently large, $v_{n}(x)$ is decreasing on $[0,1]$ and by Eq. 22, $v_{n}\left(\varepsilon_{n}\right)=\frac{\varepsilon_{n}}{n}+O\left(\frac{1}{n^{2}}\right)$, it follows from Eq. 27 that

$$
\lim _{n \rightarrow \infty} \frac{\int_{\varepsilon_{n}}^{2 \varepsilon_{n}} v_{n}}{\int_{0}^{1} v_{n}} \leq \lim _{n \rightarrow \infty} \frac{\varepsilon_{n} v_{n}\left(\varepsilon_{n}\right)}{\int_{0}^{1} v_{n}}=0 .
$$

Using once more that for $n$ sufficiently large, $v_{n}(x)$ is decreasing on [0,1], one has $v_{n}\left(2 \varepsilon_{n}\right) \leq \frac{1}{n^{2}}+O\left(\frac{1}{n^{1+\alpha}} e^{-n(1-\alpha)}\right)$ (see Eqs. 17, 21), and then infers from Eq. 27 that

$$
\lim _{n \rightarrow \infty} \frac{\int_{A_{n} \cap\left[2 \varepsilon_{n}, 1\right]} v_{n}}{\int_{0}^{1} v_{n}} \leq \lim _{n \rightarrow \infty} \frac{v_{n}\left(2 \varepsilon_{n}\right)\left|A_{n}\right|}{\int_{0}^{1} v_{n}}=0 .
$$

Similarly, one has

$$
\lim _{n \rightarrow \infty} \frac{\int_{A_{n} \cap\left[-1,-2 \varepsilon_{n}\right]} v_{n}}{\int_{0}^{1} v_{n}}=0 .
$$

To prove (ii) we obtain a lower bound for the supremum in Eq. 13 by choosing the sequence $A_{n}=\left(-\varepsilon_{n}, \varepsilon_{n}\right), n \in \mathbb{N}$. By Eq. 26 one has $\kappa=1$ in this case.

To prove (iii) we obtain a lower bound for the supremum in Eq. 13 by choosing the sequence $A_{n}=\left(-\varepsilon_{n}, \varepsilon_{n}\right), n \in \mathbb{N}$. Hence by Eq. 26 we then get

$$
\kappa \geq \kappa_{c}=\frac{c^{3} / 3}{1+c^{3} / 3} \text {. }
$$

To prove the reverse inequality we let $A_{n} \subset(-1,1), n \in \mathbb{N}$ be an arbitrary sequence of measurable sets which satisfy $\lim _{n \rightarrow \infty}\left|A_{n}\right|=0$. In view of Eq. 28 we may assume without loss of generality that $A_{n}$ is symmetric, $A_{n}=-A_{n}$, and that $\left[-\varepsilon_{n}, \varepsilon_{n}\right] \subset A_{n}$ for any $n$ (sufficiently large). It then suffices to show that

$$
\lim _{n \rightarrow \infty} \frac{\int_{A_{n} \cap\left[\varepsilon_{n}, 1\right]} v_{n}}{\int_{0}^{1} v_{n}}=0 .
$$


By Eq. 25

$$
\int_{0}^{\varepsilon_{n}} v_{n}+\int_{\varepsilon_{n}}^{1} v_{n}=\frac{1+c^{3} / 3}{n^{2}}+O\left(n^{-7 / 3}\right) .
$$

As in the proof of item (i), we estimate

$$
\int_{A_{n} \cap\left[\varepsilon_{n}, 1\right]} v_{n} \leq \int_{\varepsilon_{n}}^{2 \varepsilon_{n}} v_{n}+\int_{A_{n} \cap\left[2 \varepsilon_{n}, 1\right]} v_{n},
$$

and obtain

$$
\lim _{n \rightarrow \infty} \frac{\int_{\varepsilon_{n}}^{2 \varepsilon_{n}} v_{n}}{\int_{0}^{1} v_{n}} \leq \lim _{n \rightarrow \infty} \frac{\varepsilon_{n} v_{n}\left(\varepsilon_{n}\right)}{\int_{0}^{1} v_{n}}=0 .
$$

and

$$
\lim _{n \rightarrow \infty} \frac{\int_{A_{n} \cap\left[2 \varepsilon_{n}, 1\right]} v_{n}}{\int_{0}^{1} v_{n}} \leq \lim _{n \rightarrow \infty} \frac{v_{n}\left(2 \varepsilon_{n}\right)\left|A_{n}\right|}{\int_{0}^{1} v_{n}}=0 .
$$

Altogether we proved that $\kappa=\kappa_{c}$ and that the supremum $\kappa$ is attained by the sequence $\left(A_{n}\right)$ with $\left(-\varepsilon_{n}, \varepsilon_{n}\right)$.

In Example 2 below we analyse localisation and $\kappa$-localisation in $L^{1}$ for a family of sequences of open sets in $\mathbb{R}^{m}$, parametrised by three real positive $\alpha, \beta$ and $c$.

Example 2 Let $m \geq 1$, and let $\Omega_{n}, n \in \mathbb{N}$, be the union of $n+1$ open balls $B\left(p_{1} ; n^{-\alpha}\right), \ldots, B\left(p_{n} ; n^{-\alpha}\right), B\left(p_{n+1} ; n^{c n^{-\beta}}\right)$ with centers $p_{1}, \ldots, p_{n+1}$ respectively. Let $c>0$, and let

$$
\left|p_{i}-p_{j}\right| \geq 2+c, \quad \forall(i, j) \in\left\{\{1, \ldots, n\}^{2}, i \neq j\right\},
$$

where

$$
\beta>\alpha-\frac{1}{m} \geq 0
$$

(i) If $\beta>\alpha-\frac{1}{m+2}$, then $\left(v_{\Omega_{n}}\right)$ does not localise in $L^{1}, \kappa=0$.

(ii) If $\beta<\alpha-\frac{1}{m+2}$, then $\left(v_{\Omega_{n}}\right)$ localises in $L^{1}, \kappa=1$.

(iii) If $\beta=\alpha-\frac{1}{m+2}$, then $\left(v_{\Omega_{n}}\right) \kappa_{c}$-localises in $L^{1}$ with

$$
\kappa_{c}=\frac{c^{m+2}}{1+c^{m+2}} .
$$

An example of a sequence of open, bounded, simply connected planar sets with fixed measure 1 for which the torsion function is $\kappa$-localising in $L^{1}$ for some $0<\kappa<1$ has been given in Theorem 2 of [9].

Proof First observe that condition (29) guarantees that the $n+1$ open balls do not intersect pairwise. The first inequality in Eq. 30 guarantees that the measure of $B\left(p_{n+1} ; c n^{-\beta}\right)$ is negligible compared with the measure of $\Omega_{n}$ in the limit $n \rightarrow \infty$. The second inequality in Eq. 30 implies that $\left|\Omega_{n}\right|$ remains bounded for large $n$. The torsion function for the open ball $B(p ; R)$ is given by

Hence

$$
v_{B(p ; R)}(x)=\frac{R^{2}-|x-p|^{2}}{2 m} .
$$

$$
\left\|v_{B(p ; R)}\right\|_{1}=\rho_{m} R^{m+2},
$$


where $\rho_{m}=\frac{\omega_{m}}{m(m+2)}$ is the torsional rigidity for a ball in $\mathbb{R}^{m}$ with radius 1 . Furthermore

$$
\left\|v_{B(p ; R)}\right\|_{\infty}=\frac{R^{2}}{2 m}
$$

and

$$
\left\|v_{\Omega_{n}}\right\|_{1}=\rho_{m}\left(c^{m+2} n^{-(m+2) \beta}+n^{1-(m+2) \alpha}\right) .
$$

To prove (i) we let $A_{n} \subset \Omega_{n}, n \in \mathbb{N}$ be an arbitrary sequence of measurable sets which satisfy $\lim _{n \rightarrow \infty} \frac{\left|A_{n}\right|}{\left|\Omega_{n}\right|}=0$. We have

$$
\begin{aligned}
\int_{A_{n}} v_{\Omega_{n}} & =\int_{A_{n} \cap B\left(p_{n+1} ; c n^{-\beta}\right)} v_{\Omega_{n}}+\int_{A_{n} \cap\left(\cup_{i=1}^{n} B\left(p_{i} ; n^{-\alpha}\right)\right)} v_{\Omega_{n}} v_{\Omega_{n}}+\int_{A_{n}}\left\|v_{B\left(p_{1} ; n^{-\alpha}\right)}\right\|_{\infty} \\
& \leq \int_{B\left(p_{n+1} ; c n^{-\beta}\right)} \\
& =\rho_{m} c^{m+2} n^{-(m+2) \beta}+\frac{1}{2 m}\left|A_{n}\right| n^{-2 \alpha} .
\end{aligned}
$$

By Eq. 32 we have

$$
\begin{aligned}
\frac{\int_{A_{n}} v_{\Omega_{n}}}{\left\|v_{\Omega_{n}}\right\|_{1}} & \leq c^{m+2} n^{(m+2)(\alpha-\beta)-1}+\frac{\omega_{m}}{2 m \rho_{m}} \frac{\left|A_{n}\right|}{\left|\Omega_{n}\right|} \frac{n^{-2 \alpha}\left(c^{m} n^{-m \beta}+n^{1-m \alpha}\right)}{n^{1-(m+2) \alpha}} \\
& \leq c^{m+2} n^{(m+2)(\alpha-\beta)-1}+\frac{\omega_{m}}{2 m \rho_{m}}\left(c^{m}+1\right) \frac{\left|A_{n}\right|}{\left|\Omega_{n}\right|}
\end{aligned}
$$

where we have used Eq. 30 to bound the second term in the previous line. By the hypothesis for $\beta$ under (i) and the hypothesis on $\left(A_{n}\right)$ above we conclude

$$
\lim _{n \rightarrow \infty} \frac{\int_{A_{n}} v_{\Omega_{n}}}{\left\|v_{\Omega_{n}}\right\|_{1}}=0 .
$$

This proves the implication under Eq. 15, and concludes the proof of (i).

To prove (ii) let $A_{n}=B\left(p_{n+1} ; c n^{-\beta}\right), n \in \mathbb{N}$. This gives,

$$
\begin{aligned}
\kappa & \geq \limsup _{n \rightarrow \infty} \frac{\int_{B\left(p_{n+1} ; c n^{-\beta}\right)} v_{\Omega_{n}}}{\left\|v_{\Omega_{n}}\right\|_{1}} \\
& =\limsup _{n \rightarrow \infty} \frac{c^{m+2} n^{-(m+2) \beta}}{c^{m+2} n^{-(m+2) \beta}+n^{1-(m+2) \alpha}} .
\end{aligned}
$$

By the hypothesis for $\beta$ under (ii) we have

$$
\kappa \geq \limsup _{n \rightarrow \infty} \frac{c^{m+2} n^{-(m+2) \beta}}{c^{m+2} n^{-(m+2) \beta}+n^{1-(m+2) \alpha}}=1 .
$$

Both requirements under Eq. 14 are satisfied. This concludes the proof of (ii).

To prove (iii) we obtain a lower bound for $\kappa_{c}$ by choosing the sequence $A_{n}=$ $B\left(p_{n+1} ; c n^{-\beta}\right), n \in \mathbb{N}$. By Eq. 34 and the hypothesis for $\beta$ under (iii) we get

$$
\kappa \geq \frac{c^{m+2}}{1+c^{m+2}} .
$$


To prove the reverse inequality we let $A_{n} \subset \Omega_{n}, n \in \mathbb{N}$ be an arbitrary sequence of measurable sets which satisfy $\lim _{n \rightarrow \infty} \frac{\left|A_{n}\right|}{\left|\Omega_{n}\right|}=0$. By Eqs. 32, 33, and the hypothesis for $\beta$ under (iii),

$$
\frac{\int_{A_{n}} v_{\Omega_{n}}}{\left\|v_{\Omega_{n}}\right\|_{1}} \leq \frac{c^{m+2}}{1+c^{m+2}}+\frac{\omega_{m}}{2 m \rho_{m}} \frac{\left|A_{n}\right|}{\left|\Omega_{n}\right|}\left(c^{m} n^{-2 /(m+2)}+1\right) .
$$

By taking the $\lim \sup _{n \rightarrow \infty}$ in both sides of the inequality in Eq. 36,

$$
\limsup _{n \rightarrow \infty} \frac{\int_{A_{n}} v_{\Omega_{n}}}{\left\|v_{\Omega_{n}}\right\|_{1}} \leq \frac{c^{m+2}}{1+c^{m+2}} .
$$

By taking the supremum over all sequences $\left(A_{n}\right) \in \mathfrak{A}\left(\left(\Omega_{n}\right)\right)$ we find by Eq. 37 ,

$$
\kappa \leq \frac{c^{m+2}}{1+c^{m+2}} .
$$

This proves by Eqs. 35 and 38 that $\left(v_{\Omega_{n}}\right)$ is $\kappa_{c}$-localising with $\kappa_{c}$ given by Eq. 31 . This concludes the proof of (iii).

\section{Proof of Theorem 1}

To prove Theorem 1 we first need to establish some auxiliary results. It is well known that the torsion function can be expressed in terms of the heat kernel of $L$. Let $\Omega \subset \mathbb{R}^{m}$ be open with $0<|\Omega|<\infty$, and let $V: \Omega \rightarrow \mathbb{R}^{+}$be measurable. Denote by $p_{\Omega, V}(x, y ; t)$, $x \in \Omega, y \in \Omega, t>0$ the heat kernel of

$$
\frac{\partial u}{\partial t}=L u, \quad u \in H_{0}^{1}(\Omega) .
$$

The torsion function of $L$ then satisfies

$$
v_{\Omega, V}(x)=\int_{\Omega} d y \int_{\mathbb{R}^{+}} d t p_{\Omega, V}(x, y ; t), \quad \forall x \in \Omega .
$$

In case $V=0$ we write $p_{\Omega}$ for $p_{\Omega, V}$.

Recall the Feynman-Kac formula [22] for non-negative, measurable potentials $V: \Omega \rightarrow$ $\mathbb{R}^{+}$,

$$
\begin{aligned}
p_{\Omega, V}(x, y ; t)= & p_{\mathbb{R}^{m}}(x, y ; t) \\
& \times \mathbb{E}\left[e^{-\int_{0}^{t} V(\beta(s)) d s}\left(\Pi_{s \in[0, t]} \mathbf{1}_{\Omega}(\beta(s))\right): \beta(0)=x, \beta(t)=y\right],
\end{aligned}
$$

where $\beta$ is a Brownian bridge. Hence if $V_{1}: \Omega \rightarrow \mathbb{R}^{+}$and $V_{2}: \Omega \rightarrow \mathbb{R}^{+}$are measurable functions with $0 \leq V_{2} \leq V_{1}$, then

$$
0 \leq p_{\Omega, V_{1}}(x, y ; t) \leq p_{\Omega, V_{2}}(x, y ; t), \quad \forall x \in \Omega, \forall y \in \Omega, \forall t>0 .
$$

Since $V \geq 0$ we then conclude that

$$
0 \leq v_{\Omega, V}(x) \leq v_{\Omega}(x) \leq \mathfrak{c}_{m} \lambda_{1}(\Omega)^{-1}, \quad \forall x \in \Omega,
$$

with $\mathfrak{c}_{m}$ given by Eq. 2 .

Lemma 5 If $0<|\Omega|<\infty$, and if $V: \Omega \rightarrow \mathbb{R}^{+}$is measurable, then

$$
\lambda_{1}(\Omega, V)^{-1} \leq\left\|v_{\Omega, V}\right\|_{\infty} \leq(4+3 m \log 2) \lambda_{1}(\Omega, V)^{-1} .
$$


Lemma 5 implies that

$$
\begin{aligned}
\mathfrak{d}_{m}:= & \sup \left\{\lambda_{1}(\Omega, V)\left\|v_{\Omega, V}\right\|_{\infty}\right. \\
& \left.: \Omega \text { open in } \mathbb{R}^{m}, 0<|\Omega|<\infty, V: \Omega \rightarrow \mathbb{R}^{+}, \text {measurable }\right\}<\infty .
\end{aligned}
$$

By choosing $V=0$ in the expression under the supremum in the right-hand side of Eq. 42 we see that $\mathfrak{c}_{m} \leq \mathfrak{d}_{m}$.

Proof of Lemma 1 To prove the upper bound in Eq. 41 we note that Lemma 1 and its proof in [6] hold with $\lambda=\lambda_{1}(\Omega, V)$, and Lemma 2 and its proof in [6] hold for the semigroup associated with $L$. Finally Lemma 3 and Theorem 1 and their proofs in [6] hold with $\lambda=$ $\lambda_{1}(\Omega, V)$. This proves the upper bound in Eq. 41 . It remains to prove the lower bound. Let $\Omega_{R}=\Omega \cap B(0 ; R)$. Then $0<\left|\Omega_{R}\right| \leq \omega_{m} R^{m}$. Let $L_{R}$ be the restriction of $-\Delta+V$ acting in $L^{2}\left(\Omega_{R}\right)$ with Dirichlet boundary conditions on $\partial \Omega_{R}$. If we denote $V_{R}=\mathbf{1}_{B(p ; R)} V$, then $L_{R}$ is also the operator $-\Delta+V_{R}$ acting in $L^{2}\left(\Omega_{R}\right)$ with Dirichlet boundary conditions on $\partial \Omega_{R}$. Then $L_{R}$ is self-adjoint, and its spectrum is discrete. Since the first Dirichlet eigenfunction $\varphi_{1, \Omega_{R}, V_{R}}$ is non-negative, and $\left\|\varphi_{1, \Omega_{R}, V_{R}}\right\|_{2}=1$, one has, by the Cauchy-Schwarz inequality,

$$
0<\int_{\Omega_{R}} \varphi_{1, \Omega_{R}, V_{R}} \leq\left|\Omega_{R}\right|^{1 / 2} .
$$

By self-adjointness

$$
\begin{aligned}
\int_{\Omega_{R}} \varphi_{1, \Omega_{R}, V_{R}} & =\int_{\Omega_{R}} \varphi_{1, \Omega_{R}, V_{R}} L_{R} v_{\Omega_{R}, V_{R}}=\int_{\Omega_{R}}\left(L_{R} \varphi_{1, \Omega_{R}, V_{R}}\right) v_{\Omega_{R}, V_{R}} \\
& =\lambda_{1}\left(\Omega_{R}, V_{R}\right) \int_{\Omega_{R}} \varphi_{1, \Omega_{R}, V_{R}} v_{\Omega_{R}, V_{R}} \\
& \leq \lambda_{1}\left(\Omega_{R}, V_{R}\right)\left\|v_{\Omega_{R}, V_{R}}\right\|_{\infty} \int_{\Omega_{R}} \varphi_{1, \Omega_{R}, V_{R}} .
\end{aligned}
$$

By Eqs. 43-44 we conclude that

$$
\lambda_{1}\left(\Omega_{R}, V_{R}\right)\left\|v_{\Omega_{R}, V_{R}}\right\|_{\infty} \geq 1 .
$$

Since $\left\|v_{\Omega_{R}, V_{R}}\right\|_{\infty} \leq\left\|v_{\Omega, V}\right\|_{\infty}$ we have

$$
\lambda_{1}\left(\Omega_{R}, V_{R}\right)\left\|v_{\Omega, V}\right\|_{\infty} \geq 1 .
$$

The assertion follows since $R \mapsto \lambda_{1}\left(\Omega_{R}, V_{R}\right)$ is decreasing to $\lambda_{1}(\Omega, V)$ as $R \rightarrow \infty$.

Lemma 6 Let $\Omega \subset \mathbb{R}^{m}$ be open with $0<|\Omega|<\infty$. If $V: \Omega \rightarrow \mathbb{R}^{+}$is measurable with $0 \leq c_{1} \leq V(x) \leq c_{2}<\infty, x \in \Omega$, then

$$
v_{\Omega, c_{2}}(x) \leq v_{\Omega, V}(x) \leq v_{\Omega, c_{1}}(x),
$$

and for any $0<c<\infty, x \in \Omega$,

$$
v_{\Omega, c}(x) \geq 2^{-2(3 m+4) c / \lambda_{1}(\Omega)} \frac{\lambda_{1}(\Omega)}{8 c+\lambda_{1}(\Omega)}\left(\frac{8 c}{8 c+\lambda_{1}(\Omega)}\right)^{8 c / \lambda_{1}(\Omega)} v_{\Omega}(x) .
$$

Hence

$$
\left\|v_{\Omega, c}\right\|_{1} \geq 2^{-2(3 m+4) c / \lambda_{1}(\Omega)} \frac{\lambda_{1}(\Omega)}{8 c+\lambda_{1}(\Omega)}\left(\frac{8 c}{8 c+\lambda_{1}(\Omega)}\right)^{8 c / \lambda_{1}(\Omega)}\left\|v_{\Omega}\right\|_{1},
$$

and

$$
\left\|v_{\Omega, c}\right\|_{\infty} \geq 2^{-2(3 m+4) c / \lambda_{1}(\Omega)} \frac{\lambda_{1}(\Omega)}{8 c+\lambda_{1}(\Omega)}\left(\frac{8 c}{8 c+\lambda_{1}(\Omega)}\right)^{8 c / \lambda_{1}(\Omega)}\left\|v_{\Omega}\right\|_{\infty} .
$$


Furthermore the right-hand side of Eq. 46 converges to $v_{\Omega}(x)$ as $c \downarrow 0$.

Proof The two inequalities in Eq. 45 follow immediately from Eq. 40, and the hypothesis $0 \leq c_{1} \leq V \leq c_{2}$. To prove inequality (46), note that for any $T>0$, and any $c \geq 0$,

$$
\begin{aligned}
v_{\Omega, c}(x) & =\int_{\Omega} d y \int_{\mathbb{R}^{+}} d t p_{\Omega, c}(x, y ; t) \\
& =\int_{\Omega} d y \int_{\mathbb{R}^{+}} d t e^{-c t} p_{\Omega}(x, y ; t) \\
& \geq \int_{\Omega} d y \int_{0}^{T} d t e^{-c t} p_{\Omega}(x, y ; t) \\
& \geq e^{-c T} \int_{\Omega} d y \int_{0}^{T} d t p_{\Omega}(x, y ; t) \\
& =e^{-c T}\left(v_{\Omega}(x)-\int_{T}^{\infty} d t \int_{\Omega} d y p_{\Omega}(x, y ; t)\right) .
\end{aligned}
$$

The double integral in the right-hand side of Eq. 49 is estimated using the heat semigroup property and Tonelli's Theorem,

$$
\begin{aligned}
\int_{T}^{\infty} d t \int_{\Omega} d y p_{\Omega}(x, y ; t) & =\int_{T}^{\infty} d t \int_{\Omega} d y \int_{\Omega} d z p_{\Omega}(x, z ; t / 2) p_{\Omega}(z, y ; t / 2) \\
& \leq \int_{T}^{\infty} d t \int_{\Omega} d z p_{\Omega}(x, z ; t / 2) \int_{\Omega} d y p_{\Omega}(z, y ; t / 2) .
\end{aligned}
$$

Lemma 3 in [6] asserts that

$$
p_{\Omega}(z, y ; t) \leq(4 \pi t)^{-m / 2} 2^{m / 4} e^{-t \lambda_{1}(\Omega)-|z-y|^{2} /(8 t)} .
$$

This gives

$$
\begin{aligned}
\int_{\Omega} d y p_{\Omega}(z, y ; t) & \leq(4 \pi t)^{-m / 2} 2^{m / 4} \int_{\mathbb{R}^{m}} d y e^{-t \lambda_{1}(\Omega) / 4-|z-y|^{2} /(8 t)} \\
& =2^{3 m / 4} e^{-t \lambda_{1}(\Omega) / 4}
\end{aligned}
$$

By Eqs. 50 and 52,

$$
\begin{aligned}
\int_{T}^{\infty} d t \int_{\Omega} d y p_{\Omega}(x, y ; t) & \leq 2^{3 m / 4} \int_{T}^{\infty} d t e^{-t \lambda_{1}(\Omega) / 8} \int_{\Omega} d z p_{\Omega}(x, z ; t / 2) \\
& \leq 2^{3 m / 4} e^{-T \lambda_{1}(\Omega) / 8} \int_{\mathbb{R}^{+}} d t \int_{\Omega} d z p_{\Omega}(x, z ; t / 2) \\
& =2^{(4+3 m) / 4} e^{-T \lambda_{1}(\Omega) / 8} v_{\Omega}(x)
\end{aligned}
$$

By combining Eqs. 49 and 53 we find

$$
v_{\Omega, c}(x) \geq e^{-c T}\left(1-2^{(4+3 m) / 4} e^{-T \lambda_{1}(\Omega) / 8}\right) v_{\Omega}(x) .
$$

Choosing $T$ as to maximise the right-hand side of Eq. 54 gives that

$$
T=\frac{8}{\lambda_{1}(\Omega)} \log \left(2^{(4+3 m) / 4}\left(1+\frac{\lambda_{1}(\Omega)}{8 c}\right)\right) .
$$

Inequality Eq. 46 then follows by Eqs. 54 and 55. The inequalities Eqs. 47 and 48 follow immediately from Eq. 46. 
Proof of Theorem 1 (i) Since $0 \leq V \leq c$ we have by Eq. 45,

$$
\left\|v_{\Omega, V}\right\|_{1} \leq\left\|v_{\Omega}\right\|_{1},
$$

and

$$
\left\|v_{\Omega, V}\right\|_{\infty} \geq\left\|v_{\Omega, c}\right\|_{\infty} .
$$

The upper bound Eq. 4 follows from Eqs. 56, 57, and 48.

Similarly, by Eq. 45, we have

$$
\left\|v_{\Omega, V}\right\|_{\infty} \leq\left\|v_{\Omega}\right\|_{\infty},
$$

and

$$
\left\|v_{\Omega, V}\right\|_{1} \geq\left\|v_{\Omega, c}\right\|_{1} .
$$

The lower bound Eq. 5 follows from Eqs. 58, 59, and 47.

(ii) By using the inequality

$$
\left(1+\theta^{-1}\right)^{\theta} \leq e, \quad \forall \theta>0,
$$

with $\theta=8\left\|V_{n}\right\|_{\infty} / \lambda_{1}\left(\Omega_{n}\right)$, we obtain by Eq. 4 ,

$$
\Phi\left(\Omega_{n}, V_{n}\right) \leq e 2^{2(3 m+4) \eta}(1+8 \eta) \Phi\left(\Omega_{n}\right),
$$

and by Eq. 5 ,

$$
\Phi\left(\Omega_{n}, V_{n}\right) \geq e^{-1} 2^{-2(3 m+4) \eta}(1+8 \eta)^{-1} \Phi\left(\Omega_{n}\right) .
$$

(iii) To verify Eq. 8 we have by Lemma 4 in [10],

$$
\int_{\Omega} d y p_{\Omega}(x, y ; t) \geq 1-2^{(m+2) / 2} e^{-d_{\Omega}(x)^{2} /(8 t)} .
$$

Hence we find by Eq. 39

$$
\int_{\Omega} d x \int_{\Omega} d y p_{\Omega}(x, y ; t) \geq|\Omega|-2^{(m+2) / 2} \int_{\Omega} d x e^{-d_{\Omega}(x)^{2} /(8 t)} .
$$

Multiplying both sides of Eq. 60 by $e^{-c t}$ and integrating with respect to $t$ gives

$$
\begin{aligned}
\left\|v_{\Omega, c}\right\|_{1} & \geq \frac{|\Omega|}{c}-2^{(m+2) / 2} \int_{\Omega} d x \int_{\mathbb{R}^{+}} d t e^{-c t-d_{\Omega}(x)^{2} /(8 t)} \\
& \geq \frac{|\Omega|}{c}-2^{(m+2) / 2} \int_{\Omega} d x \int_{\mathbb{R}^{+}} d t e^{-c t / 2} \sup \left\{e^{-c t / 2-d_{\Omega}(x)^{2} /(8 t)}: t>0\right\} \\
& =\frac{|\Omega|}{c}-\frac{2^{(m+4) / 2}}{c} \int_{\Omega} d x e^{-c^{1 / 2} d_{\Omega}(x) / 2}
\end{aligned}
$$

On the other hand using Eq. 52,

$$
\begin{aligned}
\left\|v_{\Omega, c}\right\|_{\infty} & =\sup _{x \in \Omega} \int_{\mathbb{R}^{+}} d t e^{-c t} \int_{\Omega} d y p_{\Omega}(x, y ; t) \\
& \leq \sup _{x \in \Omega} \int_{\mathbb{R}^{+}} d t e^{-c t} \int_{\mathbb{R}^{m}} d y p_{\mathbb{R}^{m}}(x, y ; t) \\
& =\frac{1}{c} .
\end{aligned}
$$

The second inequality in Eq. 8 follows from Eqs. 61 and 62. Finally, Eq. 6 follows from Eq. 8, and Lebesgue's dominated convergence theorem, while Eq. 7 follows from Eq. 5 and (i). 


\section{Proof of Theorem 2}

Proof of Theorem 2. The proof follows a method from [19], which can be summarised as follows: construct a measure $\mu$ so that the efficiency of the eigenfunction of the first eigenvalue associated to $-\Delta+\mu$ almost equals 1 and then approximate $\mu$ in the sense of $\gamma$-convergence by a sequence of domains. We refer the reader to [14, Definition 4.8] (see also [12, Chapter 4]) for the notions of $\gamma$-convergence, relaxed Dirichlet problems and approximations by sequences of domains. Recall that a measure on a domain is said to be capacitary if it is nonnegative, not necessarily finite and Borel, and which is in addition absolutely continuous with respect to the capacity. One can then define a relaxed Dirichlet problem (see [14, Definition 3.1], and [12, Sections 4.3 and 3.6]). The equations $-\Delta v+$ $\mu v=1$ and $-\Delta v+\mu v=\lambda v$ can then be solved in the weak sense. The sequence of sets $\Omega_{n} \subset B_{1}$ is said to $\gamma$-convergence to the capacitary measure $\mu$ if the sequence of weak solutions $v_{n} \in H_{0}^{1}\left(\Omega_{n}\right)$ of $-\Delta v_{n}=1$ converges strongly in $L^{2}\left(B_{1}\right)$ to the weak solution $v \in H_{0}^{1}\left(B_{1}\right) \cap L^{2}(\mu)$ of $-\Delta v+\mu v=1$. As a consequence of the $\gamma$-convergence, the sequence of eigenvalues and corresponding eigenfunctions on the moving domain $\left(\Omega_{n}\right)$ converge in a suitable sense The $\gamma$-convergence is metrisable.

Let $\bar{B}_{r}$ denote the closure of $B_{r}$. For any $0<\varepsilon<1$, we consider the Dirichlet-Neumann eigenvalue problem on the annulus $A_{\varepsilon}=B_{1} \backslash \bar{B}_{1-\varepsilon}$. Denote by $\lambda_{\varepsilon}$ the first eigenvalue and by $u_{\varepsilon}$ a corresponding eigenfunction,

$$
\begin{cases}-\Delta u_{\varepsilon}=\lambda_{\varepsilon} u_{\varepsilon}, & \text { in } A_{\varepsilon}, \\ u_{\varepsilon}=0, & \text { on } \partial B_{1}, \\ \frac{\partial u_{\varepsilon}}{\partial v}=0, & \text { on } \partial B_{1-\varepsilon},\end{cases}
$$

where $v$ denotes the inward-pointing normal on the sphere $\partial B_{1-\varepsilon}$. One can show that $\lambda_{\varepsilon}$ is simple and strictly positive and that $u_{\varepsilon}$ is radially symmetric and has a constant sign, say positive. In particular, the restriction of $u_{\varepsilon}$ to $\partial B_{1-\varepsilon}$ equals a positive constant, $c_{\varepsilon}>0$. We continuously extend $u_{\varepsilon}$ inside $B_{1-\varepsilon}$ by $c_{\varepsilon}$ and denote the resulting function, defined on $\bar{B}_{1}$, by $v_{\varepsilon}$.

Since the normal derivatives of $v_{\varepsilon}$ on both sides of $\partial B_{1-\varepsilon}$ vanish, $\Delta v_{\varepsilon}$ is an $L^{2}$-function. More precisely, one has

$$
-\Delta v_{\varepsilon}=\lambda_{\varepsilon} u_{\varepsilon} 1_{A_{\varepsilon}}, \quad \text { in } \mathcal{D}^{\prime}\left(B_{1}\right),
$$

where $\mathcal{D}^{\prime}\left(B_{1}\right)$ denotes the space of distributions on $B_{1}$. By adding on both sides the $L^{2}$ function $\lambda_{\varepsilon} v_{\varepsilon} 1_{\bar{B}_{1-\varepsilon}}$ we get

$$
-\Delta v_{\varepsilon}+\lambda_{\varepsilon} v_{\varepsilon} 1_{\bar{B}_{1-\varepsilon}}=\lambda_{\varepsilon} v_{\varepsilon}, \quad \text { in } \mathcal{D}^{\prime}\left(B_{1}\right) \text {. }
$$

We view $\mu:=\lambda_{\varepsilon} 1_{\bar{B}_{1-\varepsilon}}$ as a capacitary measure on $B_{1}$. Then formally, we get

$$
-\Delta v_{\varepsilon}+\mu v_{\varepsilon}=\lambda_{\varepsilon} v_{\varepsilon}, \quad \text { in } \mathcal{D}^{\prime}\left(B_{1}\right) .
$$

Combined with the fact that $v_{\varepsilon}>0$, this means that $\lambda_{\varepsilon}$ is the first Dirichlet eigenvalue of $-\Delta+\mu$. We assume from now on that $v_{\varepsilon}$ is normalised by $\left\|v_{\varepsilon}\right\|_{L^{2}\left(B_{1}\right)}=1$. Since the measure $\mu$ is finite with support in $B_{1}$, the first eigenvalue $\lambda_{\varepsilon}$ is simple.

In view of the Dal Maso-Mosco density result [14, Theorem 4.16], there exists a sequence $\Omega_{n} \subseteq B_{1}, n \geq 1$, such that $\Omega_{n} \gamma$-converges to $\mu$. We can assume that the boundary $\partial \Omega_{n}$ of $\Omega_{n}$ is smooth. Indeed, otherwise we can replace each $\Omega_{n}$ by an inner approximation with a smooth open set, which is close enough in the sense of the distance associated to the $\gamma$ convergence. Furthermore, we can also assume that $\Omega_{n}$ is connected. Indeed, since $\Omega_{n}$ is smooth, it has a finite number of smooth connected components, which are separated from 
each other by a positive distance. These components can be joined by a finite number of thin tubes, connecting the set $\Omega_{n}$. As the width of the tubes vanishes, the sequence $\gamma$-converges by Sverak's theorem (see [12, Theorem 4.7.1]).

It is possible to explicitly construct such a sequence $\left(\Omega_{n}\right)$ in the spirit of CioranescuMurat [13], but such a construction is not needed for the rest of the proof. The only fact we need to keep in mind is that $\Omega_{n} \subseteq B_{1}$, so that $\left|\Omega_{n}\right| \leq\left|B_{1}\right|$. Denote by $\lambda_{1}\left(\Omega_{n}\right)$ the first Dirichlet eigenvalue of $-\Delta$ on $\Omega_{n}$ and by $u_{n}$ the $L^{2}$-normalised, positive eigenfunction corresponding to $\lambda_{1}\left(\Omega_{n}\right)$. We extend $u_{n}$ to $B_{1}$ by setting it to 0 on $B_{1} \backslash \bar{\Omega}_{n}$ and by a slight abuse of notation, denote this extension again by $u_{n}$. The $\gamma$-convergence of $\left(\Omega_{n}\right)$, together with the compact embedding of $H_{0}^{1}\left(B_{1}\right)$ in $L^{1}\left(B_{1}\right)$, imply that (i) $\lambda_{1}\left(\Omega_{n}\right) \rightarrow \lambda_{\varepsilon}$, and (ii) $u_{n} \rightarrow v_{\varepsilon}$ weakly in $H_{0}^{1}\left(B_{1}\right)$ and strongly in $L^{1}\left(B_{1}\right)$. Assuming that

$$
\lim _{n \rightarrow \infty}\left\|u_{n}\right\|_{\infty}=\left\|v_{\varepsilon}\right\|_{\infty}
$$

we get

$$
\liminf _{n \rightarrow+\infty} \frac{\int_{\Omega_{n}} u_{n}}{\left\|u_{n}\right\|_{\infty}\left|\Omega_{n}\right|} \geq \frac{\int_{B_{1}} v_{\varepsilon}}{\left\|v_{\varepsilon}\right\|_{\infty}\left|B_{1}\right|} .
$$

Since the right-hand side is arbitrarily close to 1 when $\varepsilon \downarrow 0$, the proof of Theorem 2 is then completed by a diagonal selection procedure.

It remains to show Eq. 63. This kind of assertion is known to be true in a general setup. In essence it is a consequence of the subharmonicity of the eigenfunctions $u_{n}, n \geq 1$. (For a similar result for the torsion function see [19, Theorem 2.2].) For the sake of completeness, we give below a proof. It slightly differs from the one in [19, Theorem 2.2].

First note that since $u_{n} \rightarrow v_{\varepsilon}$ strongly in $L^{1}$, it follows that

$$
\liminf _{n \rightarrow+\infty}\left\|u_{n}\right\|_{\infty} \geq\left\|v_{\varepsilon}\right\|_{\infty} .
$$

To prove that lim $\sup _{n \rightarrow+\infty}\left\|u_{n}\right\|_{\infty} \leq\left\|v_{\varepsilon}\right\|_{\infty}$ we argue as follows. Being convergent, the sequence $\left(\lambda_{1}\left(\Omega_{n}\right)\right)$ is bounded, and so is $\left\|u_{n}\right\|_{\infty}$. Choose $M>0$ so that for any $n \in \mathbb{N}$

$$
\lambda_{1}\left(\Omega_{n}\right) u_{n}(x) \leq M, \quad \forall x \in B_{1},
$$

and therefore

$$
-\Delta u_{n}(x) \leq M, \quad \forall x \in B_{1} .
$$

Let $x_{n} \in B_{1}$ be a maximum point for $u_{n}$. By taking, if necessary, a subsequence we may assume that $x_{n} \rightarrow x^{*}$. Furthermore,

$$
-\Delta u_{n} \leq M=M \Delta \frac{\left|x-x_{n}\right|^{2}}{2 m}, \quad \text { in } \mathcal{D}^{\prime}\left(\mathbb{R}^{m}\right),
$$

or

$$
-\Delta\left(u_{n}+M \frac{\left|x-x_{n}\right|^{2}}{2 m}\right) \leq 0, \quad \text { in } \mathcal{D}^{\prime}\left(\mathbb{R}^{m}\right) .
$$

By the subharmonicity of the function $x \mapsto u_{n}(x)+M \frac{\left|x-x_{n}\right|^{2}}{2 m}$ around $x_{n}$, it then follows that for $\delta>0$ sufficiently small,

$$
\left\|u_{n}\right\|_{\infty}=u_{n}\left(x_{n}\right) \leq \frac{\int_{B\left(x_{n} ; \delta\right)} d x\left(u_{n}(x)+M \frac{\left|x-x_{n}\right|^{2}}{2 m}\right)}{\left|B\left(x_{n} ; \delta\right)\right|} .
$$

Taking the limit $n \rightarrow+\infty$ we obtain

$$
\limsup _{n \rightarrow+\infty}\left\|u_{n}\right\|_{\infty} \leq \frac{\int_{B\left(x^{*} ; \delta\right)} d x\left(v_{\varepsilon}(x)+M \frac{\left|x-x^{*}\right|^{2}}{2 m}\right)}{\left|B\left(x^{*} ; \delta\right)\right|},
$$


or

$$
\limsup _{n \rightarrow+\infty}\left\|u_{n}\right\|_{\infty} \leq \frac{\left\|v_{\varepsilon}\right\|_{\infty}\left|B\left(x^{*} ; \delta\right)\right|+M \frac{\delta^{2}}{2 m}\left|B\left(x^{*} ; \delta\right)\right|}{\left|B\left(x^{*} ; \delta\right)\right|}=\left\|v_{\varepsilon}\right\|_{\infty}+M \frac{\delta^{2}}{2 m} .
$$

Letting $\delta \downarrow 0$, completes the proof.

Remark 1 If $\Omega$ is an open connected subset of $\mathbb{R}^{m}$ with $m \geq 2$ and $0<|\Omega|<\infty$, then

$$
\Phi(\Omega) \geq \frac{E(\Omega)}{1+k_{m} \lambda_{1}(\Omega)^{m / 4}|\Omega|^{1 / 2}(1-E(\Omega))^{1 / 2}}, m=2,3,
$$

(ii)

$$
\Phi(\Omega) \geq \frac{E(\Omega)}{1+k_{m} \lambda_{1}(\Omega)|\Omega|^{2 / m}(1-E(\Omega))^{1 /(m-1)}}, \quad m \geq 4,
$$

where

$$
k_{m}= \begin{cases}2(8 \pi)^{-m / 4} \Gamma((4-m) / 4), & m=2,3, \\ \pi^{-1}(m-2)^{-1} m^{-1 /(m-1)}(\Gamma((m+2) / 2))^{2 / m}, & m \geq 4 .\end{cases}
$$

Proof Putting $V=0$ in Eq. 39 one obtains by using Eq. 51,

$$
\begin{aligned}
v_{\Omega}(x) & \geq \int_{\Omega} d y \int_{\mathbb{R}^{+}} d t p_{\Omega}(x, y ; t) \frac{\varphi_{1, \Omega}(y)}{\left\|\varphi_{1, \Omega}\right\|_{\infty}} \\
& =\lambda_{1}(\Omega)^{-1} \frac{\varphi_{1, \Omega}(x)}{\left\|\varphi_{1, \Omega}\right\|_{\infty}} .
\end{aligned}
$$

Integrating both sides of Eq. 67 yields,

$$
\left\|v_{\Omega}\right\|_{1} \geq \lambda_{1}(\Omega)^{-1} \frac{\left\|\varphi_{1, \Omega}\right\|_{1}}{\left\|\varphi_{1, \Omega}\right\|_{\infty}} .
$$

To obtain the stated lower bound for $\Phi(\Omega)$ it remains to find an upper bound for $\left\|v_{\Omega}\right\|_{\infty}$.

First consider case (i). By Eq. 67, the Cauchy-Schwarz inequality, and the heat semigroup property, one sees that

$$
\begin{aligned}
v_{\Omega}(x)-\lambda_{1}(\Omega)^{-1} \frac{\varphi_{1, \Omega}(x)}{\left\|\varphi_{1, \Omega}\right\|_{\infty}} & =\int_{\Omega} d y \int_{\mathbb{R}^{+}} d t p_{\Omega}(x, y ; t)\left(1-\frac{\varphi_{1, \Omega}(y)}{\left\|\varphi_{1, \Omega}\right\|_{\infty}}\right) \\
& \leq \int_{\mathbb{R}^{+}} d t\left(\int_{\Omega} d y\left(p_{\Omega}(x, y ; t)\right)^{2}\right)^{1 / 2}\left(\int_{\Omega} d y\left(1-\frac{\varphi_{1, \Omega}(y)}{\left\|\varphi_{1, \Omega}\right\|_{\infty}}\right)\right)^{1 / 2} \\
& =|\Omega|^{1 / 2} \int_{\mathbb{R}^{+}} d t p_{\Omega}(x, x ; 2 t)^{1 / 2}(1-E(\Omega))^{1 / 2}
\end{aligned}
$$

Choosing $\beta=\frac{1}{2}$ in Lemma 1 of [6] gives by domain monotonicity of the Dirichlet heat kernel, and Eq. 52

$$
p_{\Omega}(x, x ; 2 t) \leq e^{-t \lambda_{1}(\Omega)}(4 \pi t)^{-m / 2} .
$$

Substitution of Eq. 70 into the right-hand side of Eq. 69, evaluating the resulting integral with respect to $t$, and taking the supremum over all $x \in \Omega$ gives

$$
\left\|v_{\Omega}\right\|_{\infty}-\lambda_{1}(\Omega)^{-1} \leq 2(8 \pi)^{-m / 4}|\Omega|^{1 / 2} \Gamma((4-m) / 4) \lambda_{1}(\Omega)^{-1+m / 4}(1-E(\Omega))^{1 / 2} .
$$

Inequality Eq. 64 follows from Eqs. 68, Eq. 71 with the values for $k_{2}$ and $k_{3}$ given in Eq. 66. 
Next consider case (ii). By the first equality in Eq. 69 we have by domain monotonicity of the Dirichlet heat kernel

$$
\begin{aligned}
v_{\Omega}(x)-\lambda_{1}(\Omega)^{-1} \frac{\varphi_{1, \Omega}(x)}{\left\|\varphi_{1, \Omega}\right\|_{\infty}} & \leq \int_{\Omega} d y \int_{\mathbb{R}^{+}} d t p_{\mathbb{R}^{m}}(x, y ; t)\left(1-\frac{\varphi_{1, \Omega}(y)}{\left\|\varphi_{1, \Omega}\right\|_{\infty}}\right) \\
& =c_{m} \int_{\Omega} d y|x-y|^{2-m}\left(1-\frac{\varphi_{1, \Omega}(y)}{\left\|\varphi_{1, \Omega}\right\|_{\infty}}\right),
\end{aligned}
$$

where

$$
c_{m}=\frac{\Gamma((m-2) / 2)}{4 \pi^{m / 2}} .
$$

By Hölder's inequality with exponents $p=\frac{m-1}{m-2}$ and $q=m-1$ we have

$$
\begin{aligned}
& v_{\Omega}(x) \lambda_{1}(\Omega)^{-1} \frac{\varphi_{1, \Omega}(x)}{\left\|\varphi_{1, \Omega}\right\|_{\infty}} \\
\leq & c_{m}\left(\int_{\Omega} \frac{d y}{|x-y|^{m-1}}\right)^{(m-2) /(m-1)}\left(\int_{\Omega} d y\left(1-\frac{\varphi_{1, \Omega}(y)}{\left\|\varphi_{1, \Omega}\right\|_{\infty}}\right)\right)^{1 /(m-1)} \\
\leq & c_{m}\left(\int_{\Omega^{*}} \frac{d y}{|y|^{m-1}}\right)^{(m-2) /(m-1)}\left(\int_{\Omega} d y\left(1-\frac{\varphi_{1, \Omega}(y)}{\left\|\varphi_{1, \Omega}\right\|_{\infty}}\right)\right)^{1 /(m-1)} \\
= & c_{m}\left(m \omega_{m} R^{*}\right)^{(m-2) /(m-1)}|\Omega|^{1 /(m-1)}(1-E(\Omega))^{1 /(m-1)},
\end{aligned}
$$

where we have used Schwarz symmetrisation with $\Omega^{*}=B_{R^{*}}$, and $\omega_{m}\left(R^{*}\right)^{m}=|\Omega|$.

Taking the supremum over all $x \in \Omega$ and using the formulae for $c_{m}$ and $R^{*}$ gives,

$$
\begin{aligned}
& \left\|v_{\Omega}\right\|_{\infty} \leq \lambda_{1}(\Omega)^{-1}+\pi^{-1}(m-2)^{-1} m^{-1 /(m-1)}(\Gamma((m+2) / 2))^{2 / m} \\
& \times|\Omega|^{2 / m}(1-E(\Omega))^{1 /(m-1)} .
\end{aligned}
$$

This, together with Eq. 68, implies the assertion for $m \geq 4$.

We see from the proof of Remark 1(ii) that the case $m=3$ could also have been included. However, that would have given $\lambda_{1}(\Omega)|\Omega|^{2 / 3}$ in the denominator. By Theorem 3 (iii) we have, for $m=3$, that $\lambda_{1}(\Omega)|\Omega|^{2 / 3} \gg 1$ if $E(\Omega)$ is close to 1 . Then $\lambda_{1}(\Omega)^{3 / 4}|\Omega|^{1 / 2} \ll$ $\lambda_{1}(\Omega)|\Omega|^{2 / 3}$, and so Eq. 64 gives a better bound in that case. However, bounds (64) and (65) do not imply that if $\Phi(\Omega)$ is close to 1 then $E(\Omega)$ is close to 1 since, by Theorem 3 (iii), $\lambda_{1}(\Omega)|\Omega|^{2 / m}$ becomes large.

\section{Proof of Theorem 3}

Proof of Theorem 3(i). Since $\|u\|_{\infty}>0$ we can re-scale both $u$ and $\Omega$, such that $\|u\|_{\infty}=1$, and $|\Omega|=\omega_{m}$. Inequality (9) then reads

$$
\left(1-f_{\Omega} u\right) \int_{\Omega}|\nabla u|^{2} \geq \frac{4 m^{2}}{(m+2)^{2}} \omega_{m},
$$

with $|\Omega|=\omega_{m},\|u\|_{\infty}=1$, and $f_{\Omega} u \geq \frac{2}{m+2}$. Note that replacing $u$ by its positive part $u^{+}$ decreases the left-hand side of Eq. 72, and furthermore, $u^{+} \leq 1, f_{\Omega} u^{+} \geq \frac{2}{m+2}$. So it suffices to prove that for any $m \geq 2$,

$$
(1-\theta) F(\theta) \geq \frac{4 m^{2}}{(m+2)^{2}} \omega_{m}, \quad \forall \theta \in[2 /(m+2), 1),
$$


where

$$
F(\theta):=\inf \left\{\int_{\Omega}|\nabla u|^{2}: u \in H_{0}^{1}(\Omega), 0 \leq u \leq 1, f_{\Omega} u=\theta\right\}
$$

We make some preliminary observations. By Schwarz rearrangement we may consider the infimum in the definition of $F$ over the collection $H_{0}^{* 1}\left(B_{1}\right)$ of all radially symmetric, decreasing functions $u$ in $H_{0}^{1}\left(B_{1}\right)$ since this rearrangement decreases the energy and leaves the other constraints unchanged. So,

$$
F(\theta) \geq F^{*}(\theta), \quad 2 /(m+2) \leq \theta<1,
$$

where

$$
F^{*}(\theta)=\inf \left\{\int_{B_{1}}|\nabla u|^{2}: u \in H_{0}^{* 1}\left(B_{1}\right), 0 \leq u \leq 1, f_{B_{1}} u=\theta\right\}
$$

First note that

$$
\inf \left\{\int_{B_{1}}|\nabla u|^{2}: u \in H_{0}^{* 1}\left(B_{1}\right), f_{B_{1}} u=\theta\right\}
$$

admits a minimiser. By the Lagrange multiplier theorem, there exists a constant $c$ such that

$$
-\Delta u=c, \quad u \in H_{0}^{* 1}\left(B_{1}\right), \quad f_{B_{1}} u=\theta,
$$

Since $u$ is radially symmetric and decreasing, in the sequel, by a slight abuse of notation, we write $u(r)$ instead of $u(x)$. By a straightforward computation one sees that for any $2 /(m+$ 2) $\leq \theta<1$

$$
u(r)=2^{-1}(m+2) \theta\left(1-r^{2}\right)
$$

In particular, $\|u\|_{\infty}=u(0) \geq 1$. Note that we could have written $0 \leq u,\|u\|_{\infty}=1$ instead of $0 \leq u \leq 1$ in the right-hand side of Eq. 73 .

For any $2 /(m+2) \leq \theta<1, F^{*}(\theta)$ admits a minimiser. Since the Dirichlet energy is strictly convex, it is unique, and we denote it by $u_{\theta}$. Let

$$
f(\theta)=(1-\theta) F^{*}(\theta) .
$$

Since $F(\theta) \geq F^{*}(\theta)$, it suffices to show that

$$
f(\theta) \geq \frac{4 m^{2}}{(m+2)^{2}} \omega_{m}, \quad \forall \theta \in[2 /(m+2), 1) .
$$

If $\theta=\frac{2}{m+2}$, then by Eq. 74, $u_{2 /(m+2)}(r)=1-r^{2}$. Hence

$$
f(2 /(m+2))=\left(1-\frac{2}{m+2}\right) \int_{B_{1}}(-2 r)^{2} m r^{m-1} \omega_{m} d r=\frac{4 m^{2}}{(m+2)^{2}} \omega_{m} .
$$

Hence (75) is satisfied for $\theta=\frac{2}{m+2}$. 
The remaining part of the proof consists of four parts. In part (a) we show that Eq. 75 holds for any $m \geq 2$ and $\theta \in\left[\frac{2}{m+2}, \theta_{m}^{*}\right]$ where

$$
\theta_{m}^{*}:=\frac{m+\left(m^{2}+8 m\right)^{1 / 2}}{2(m+2)} .
$$

We note that $2 /(m+2)<2 / 3<\theta_{m}^{*}<1$ for any $m \geq 2$. In part (b) we prove that Eq. 75 holds for any $m \geq 4$ and $\theta \in\left[\theta_{m}^{*}, 1\right)$. In part (c) we show that Eq. 75 holds for $m=2,3$, using the Euler-Lagrange equation of a variational problem, related to an obstacle problem. See Eq. 96 below. Finally, in part (d) we verify that equality in Eq. 9 holds if and only if $\Omega$ is a ball and $u$ is a multiple of the torsion function for that ball. This completes the proof of Theorem 3 (i).

(a) Assume that $\frac{2}{m+2} \leq \theta \leq \theta_{m}^{*}$. In a first step we prove that $\theta \mapsto f(\theta)$ is increasing on $\left[\frac{2}{m+2}, \frac{2}{3}\right]$ which by Eq. 76 implies that Eq. 75 holds for $\theta$ in the latter interval. Given $\frac{2}{m+2}<\theta<1$, choose any $\varepsilon>0$ with the property

$$
\frac{\theta}{1+\varepsilon}>\frac{2}{m+2} \text {. }
$$

Let $\bar{u}_{\theta}$ be the minimiser of the obstacle problem

$$
\inf \left\{\int_{B_{1}}|\nabla u|^{2}: u \in H_{0}^{* 1}\left(B_{1}\right), 0 \leq u \leq 1+\varepsilon, f_{B_{1}} u=\theta\right\} .
$$

Then, by inclusion of the class of admissible test functions,

$$
\int_{B_{1}}\left|\nabla \bar{u}_{\theta}\right|^{2} \leq \int_{B_{1}}\left|\nabla u_{\theta}\right|^{2}
$$

where $u_{\theta}$ is the minimiser of $F^{*}(\theta)$. Furthermore $(1+\varepsilon)^{-1} \bar{u}_{\theta}$ is the minimizer $u_{\theta /(1+\varepsilon)}$ for $F^{*}(\theta /(1+\varepsilon))$, since $0 \leq(1+\varepsilon)^{-1} \bar{u}_{\theta} \leq 1$, and $f_{B_{1}} \frac{\bar{u}_{\theta}}{1+\varepsilon}=\frac{\theta}{1+\varepsilon}$. We wish to prove that

$$
f(\theta /(1+\varepsilon))=\left(1-\frac{\theta}{1+\varepsilon}\right) \int_{B_{1}} \frac{\left|\nabla \bar{u}_{\theta}\right|^{2}}{(1+\varepsilon)^{2}} \leq(1-\theta) \int_{B_{1}}\left|\nabla u_{\theta}\right|^{2}=f(\theta),
$$

By Eq. 78, inequality (79) holds if

$$
\frac{1}{(1+\varepsilon)^{2}}\left(1-\frac{\theta}{1+\varepsilon}\right) \leq(1-\theta),
$$

or, after simplifying,

$$
1 \leq\left(3+3 \varepsilon+\varepsilon^{2}\right)(1-\theta) .
$$

The latter inequality clearly holds for any $\varepsilon \geq 0$ if $\frac{2}{m+2} \leq \theta \leq \frac{2}{3}$. Thus we have proved that $f(\theta)$ is increasing on the interval $\left[\frac{2}{m+2}, \frac{2}{3}\right]$.

By the same argument we now prove that $f(\theta) \geq f(2 /(m+2))=\frac{4 m^{2}}{(m+2)^{2}} \omega_{m}$ also for $\theta \in\left(\frac{2}{3}, \theta_{m}^{*}\right]$. (However, we do not prove that $f$ is increasing on the interval $\left(\frac{2}{3}, \theta_{m}^{*}\right]$.) Given $\theta \in\left(\frac{2}{3}, \theta_{m}^{*}\right]$, we want to find $\varepsilon>0$ so that

$$
\frac{2}{m+2} \leq \frac{\theta}{1+\varepsilon} \leq \frac{2}{3}, \quad 1 \leq\left(3+3 \varepsilon+\varepsilon^{2}\right)(1-\theta) .
$$

By Eqs. 79 and 80, one then infers that

$$
f(\theta) \geq f(\theta /(1+\varepsilon)) \geq f(2 /(m+2)) .
$$


To find $\varepsilon>0$ so that Eq. 81 holds, we solve

$$
1=3(1-\theta)+3 \varepsilon(1-\theta)+\varepsilon^{2}(1-\theta)
$$

for $\varepsilon$, and verify that for the given range of $\theta$, one has $\frac{2}{m+2} \leq \frac{\theta}{1+\varepsilon} \leq \frac{2}{3}$. The positive solution of Eq. 82 is given by

$$
\varepsilon=-\frac{3}{2}+\frac{1}{2}\left(\frac{1+3 \theta}{1-\theta}\right)^{1 / 2}>0
$$

Since

$$
\theta \leq \theta_{m}^{*}
$$

we have that the requirement $\frac{2}{m+2} \leq \frac{\theta}{1+\varepsilon}$ is fulfilled. Using $\theta \geq \frac{2}{3}$, one sees that $\frac{\theta}{1+\varepsilon} \leq \frac{2}{3}$.

(b) In this part we assume that $m \geq 4$ and $\theta_{m}^{*} \leq \theta<1$. Let $0 \leq r_{0}<1$ and let $u \in H_{0}^{* 1}\left(B_{1}\right)$ with $0 \leq u \leq 1$ and $\theta=f_{B_{1}} u$. Since $u$ is radially symmetric and decreasing,

$$
\omega_{m}^{-1} \int_{B_{1} \backslash \bar{B}_{r_{0}}} u \leq \omega_{m}^{-1} u\left(r_{0}\right)\left|B_{1} \backslash \bar{B}_{r_{0}}\right|=u\left(r_{0}\right)\left(1-r_{0}^{m}\right) .
$$

Since, $0 \leq u \leq 1$ and $\theta=f_{B_{1}} u$, we conclude that

$$
r_{0}^{m} \geq \omega_{m}^{-1} \int_{B_{r_{0}}} u=\omega_{m}^{-1} \int_{B_{1}} u-\omega_{m}^{-1} \int_{B_{1} \backslash \bar{B}_{r_{0}}} u \geq \theta-u\left(r_{0}\right)\left(1-r_{0}^{m}\right) .
$$

It then follows that

$$
u\left(r_{0}\right) \geq \eta_{m}\left(\theta, r_{0}\right):=\frac{\theta-r_{0}^{m}}{1-r_{0}^{m}} \geq 0, \quad \forall r_{0} \in\left[0, \theta^{1 / m}\right] .
$$

By inclusion of the admissible test functions one has

$$
f(\theta) \geq(1-\theta) \inf \left\{\int_{B_{1}}|\nabla u|^{2}: u \in H_{0}^{* 1}\left(B_{1}\right), u\left(r_{0}\right) \geq \eta_{m}\left(\theta, r_{0}\right)\right\} .
$$

The infimum in the right-hand side of Eq. 84 is attained and its minimiser $u^{*}$ is given by

$$
u^{*}(r)=\left\{\begin{array}{lr}
\eta_{m}\left(\theta, r_{0}\right), & 0 \leq r \leq r_{0} \\
\frac{1-r^{2-m}}{1-r_{0}^{2-m}} \eta_{m}\left(\theta, r_{0}\right), & r_{0} \leq r \leq 1
\end{array}\right.
$$

A straightforward calculation gives

$$
\int_{B_{1}}\left|\nabla u^{*}\right|^{2}=m(m-2) \omega_{m} \frac{\eta_{m}^{2}\left(\theta, r_{0}\right)}{r_{0}^{2-m}-1} .
$$

We now choose

$$
r_{0}=\theta^{c / m},
$$

where $c \geq 1$ is to be determined later. This choice satisfies the constraint $0 \leq r_{0} \leq \theta^{1 / m}$ in Eq. 83. By Eqs. 85 and 86,

$$
f(\theta) \geq m(m-2) \omega_{m}\left(\frac{\theta-\theta^{c}}{1-\theta^{c}}\right)^{2} \frac{1-\theta}{\theta^{c(2-m) / m}-1} .
$$

By L'Hôpital's rule,

$$
\liminf _{\theta \uparrow 1} f(\theta) \geq m^{2} \omega_{m} \frac{(c-1)^{2}}{c^{3}} .
$$


The right-hand side of Eq. 88 is maximised for $c=3$. This choice yields,

$$
\liminf _{\theta \uparrow 1} f(\theta) \geq \frac{4 m^{2}}{27} \omega_{m}
$$

Note that

$$
\frac{4 m^{2}}{27} \omega_{m} \geq \frac{4 m^{2}}{(m+2)^{2}} \omega_{m} .
$$

if and only if $m \geq 4$. This is why the proof of Eq. 75 for $m=2,3$ has been deferred to part (c). By Eq. 87 we have for $c=3$,

$$
\begin{aligned}
f(\theta) & \geq m(m-2) \omega_{m}\left(\frac{\theta(1+\theta)}{1+\theta+\theta^{2}}\right)^{2} \frac{1-\theta}{\theta^{3(2-m) / m}-1} \\
& \geq m(m-2) \omega_{m}\left(\frac{\theta(1+\theta)}{1+\theta+\theta^{2}}\right)^{2} \frac{1-\theta}{\theta^{-3}-1} \\
& =m(m-2) \omega_{m} \frac{\theta^{5}(1+\theta)^{2}}{\left(1+\theta+\theta^{2}\right)^{3}} .
\end{aligned}
$$

To prove (75) for $\theta \in\left[\theta_{m}^{*}, 1\right)$ and $m \geq 4$, it suffices, by Eq. 90, to show that

$$
\frac{\theta^{5}(1+\theta)^{2}}{\left(1+\theta+\theta^{2}\right)^{3}} \geq \frac{4 m}{(m-2)(m+2)^{2}}, \quad \forall \theta \in\left[\theta_{m}^{*}, 1\right) .
$$

First observe that the left-hand side of Eq. 91 is a product of non-negative increasing functions, $\theta \mapsto \frac{\theta(1+\theta)}{1+\theta+\theta^{2}}$ and $\theta \mapsto \frac{\theta^{3}}{1+\theta+\theta^{2}}$, and so is increasing. So if Eq. 91 holds for $\theta=\theta_{m}^{*}$ then it holds on the interval $\left[\theta_{m}^{*}, 1\right)$. Furthermore by Eq. 77 ,

$$
\theta_{m}^{*}=1-\frac{8}{(m+2)\left(m+4+\left(m^{2}+8 m\right)^{1 / 2}\right)} .
$$

Hence $\left(\theta_{m}^{*}\right)_{m}$ is a strictly increasing sequence. Since the right-hand side of Eq. 91 is decreasing in $m$ we conclude that if Eq. 91 holds for $m=m_{1}$ then it holds for all $m \geq m_{1}$. It is straightforward to verify

$$
\theta_{6}^{*}>\frac{15}{16}
$$

and that Eq. 91 holds for $\theta=\frac{15}{16}$, and $m=6$. Hence it follows that Eq. 91 holds for all $\theta \in\left[\theta_{m}^{*}, 1\right)$ with $m \geq 6$.

To complete the proof of part (b) it remains to treat the cases $m=4$ and $m=5$. We first consider the case $m=4$. One computes

$$
\theta_{4}^{*}=\frac{1+\sqrt{3}}{3}
$$

and by the first inequality in Eq. 90, one gets

$$
f(\theta) \geq 8 \omega_{4}\left(\frac{\theta(1+\theta)}{1+\theta+\theta^{2}}\right)^{2}\left(\frac{1-\theta}{\theta^{-3 / 2}-1}\right) .
$$

Since both $\theta \mapsto \frac{\theta(1+\theta)}{1+\theta+\theta^{2}}$ and $\theta \mapsto \frac{1-\theta}{\theta^{-3 / 2}-1}$ are non-negative increasing functions on the interval $[0,1)$ the right-hand side of Eq. 92 is increasing in $\theta$. Note that the right-hand side of Eq. 89 equals $16 \omega_{4} / 9$. Hence by Eq. 92 it suffices to verify that

$$
\left(\frac{\theta_{4}^{*}\left(1+\theta_{4}^{*}\right)}{1+\theta_{4}^{*}+\theta_{4}^{* 2}}\right)^{2}\left(\frac{1-\theta_{4}^{*}}{\theta_{4}^{*-3 / 2}-1}\right) \geq \frac{2}{9} .
$$


Numerical evaluation of the left-hand side of Eq. 93 yields

$$
\left(\frac{\theta_{4}^{*}\left(1+\theta_{4}^{*}\right)}{1+\theta_{4}^{*}+\theta_{4}^{* 2}}\right)^{2}\left(\frac{1-\theta_{4}^{*}}{\theta_{4}^{*-3 / 2}-1}\right) \geq .238,
$$

which implies (93).

Finally we consider the case $m=5$. One computes that

$$
\theta_{5}^{*} \geq \frac{13}{14}
$$

and by the first inequality in Eq. 90,

$$
f(\theta) \geq 15 \omega_{5}\left(\frac{\theta(1+\theta)}{1+\theta+\theta^{2}}\right)^{2} \frac{1-\theta}{\theta^{-9 / 5}-1} .
$$

Since both $\theta \mapsto \frac{\theta(1+\theta)}{1+\theta+\theta^{2}}$ and $\theta \mapsto \frac{1-\theta}{\theta^{-9 / 5}-1}$ are non-negative increasing functions on the interval $[0,1)$, so is the right-hand side of Eq. 92 and it remains, by Eq. 92, to verify that

$$
\left(\frac{\theta_{5}^{*}\left(1+\theta_{5}^{*}\right)}{1+\theta_{5}^{*}+\theta_{5}^{* 2}}\right)^{2}\left(\frac{1-\theta_{5}^{*}}{\theta_{5}^{*-9 / 5}-1}\right) \geq \frac{20}{147} .
$$

By Eq. 94, the left-hand side of Eq. 95 is bounded from below by .206 while the right hand side of Eq. 95 is bounded from above by .137. This completes the proof of part (b).

(c) In this part we treat the cases $m=2$ and $m=3$. We begin with some preliminary considerations. We note that the minimisation problem (73) is related to a volume constraint obstacle problem in $B_{1}$ : we claim that there exist $c>0$ and $0 \leq l<1$, depending on $\theta$, so that $u_{\theta}$ satisfies the following system of equations,

$$
\begin{cases}-\Delta u=c, & \text { in } B_{1} \backslash \bar{B}_{l}, \\ u=1, & \text { on } \bar{B}_{l}, \\ u=0, & \text { on } \partial B_{1}, \\ \frac{\partial u}{\partial v}=0, & \text { on } \partial B_{l},\end{cases}
$$

where $v$ denotes the inward pointing normal on the sphere $\partial B_{l}$. Indeed, since $u_{\theta}$ is radially symmetric, decreasing and since $u_{\theta}(0)=1$ (see Eq. 73) and $u_{\theta}(1)=0$, there exists a maximal number $0 \leq l \equiv l(\theta)<1$ so that $u_{\theta}(r)=1$ for $0 \leq r \leq l$. By the Lagrange multiplier theorem, there exists a constant $c>0$ so that $-\Delta u_{\theta}=c$ on $B_{1} \backslash \bar{B}_{l}$ in the sense of distributions. It then follows from [16, Theorem 2] that $u_{\theta}$ is $C^{1, \alpha}$ on $\bar{B}_{1}$ which implies that $\frac{\partial u_{\theta}}{\partial v}=0$ on $\partial B_{l}$. These observations establish (96). Note that both $c$ and $l$ are uniquely determined by $\theta$.

We claim that the map

$$
\mathfrak{b}:\left[\frac{2}{m+2}, 1\right) \rightarrow[0,1), \theta \mapsto l(\theta),
$$

is an increasing bijection. To prove the latter assertion, we construct for any given $0 \leq$ $l<1$ a unique radially symmetric, decreasing solution $u(\cdot ; l)$ of Eq. 96 and show that $\theta \equiv \theta(l):=f_{B_{1}} u(\cdot ; l)$, satisfies $2 /(m+2) \leq \theta<1$ with $\theta(0)=2 /(m+2)$ and $\lim _{l \rightarrow 1} \theta(l)=1$. First we note that $c$ is uniquely determined by $l$ since for any given 
$0 \leq l<1$, the solution of Eq. 96 is given by a formula. To obtain it, note that the general radially symmetric solution of $-\Delta u=c$ on the annulus $B_{1} \backslash \bar{B}_{l}$ is of the form

$$
u(r)= \begin{cases}-c \frac{r^{2}}{4}+a \ln (r)+b & \text { if } m=2, \\ -c \frac{r^{2}}{2 m}-\frac{a}{(m-2) r^{m-2}}+b & \text { if } m \geq 3\end{cases}
$$

for some real constants $a, b, c$. The condition $\frac{\partial u}{\partial v}(l)=0$ implies that $a=\frac{l^{m} c}{m}$ so that the boundary condition $u(1)=0$ leads to

$$
u(r)=\left\{\begin{array}{lc}
\frac{c}{4}\left(1-r^{2}\right)+\frac{l^{2} c}{2} \ln (r) & \text { if } m=2, \\
\frac{c}{2 m}\left(1-r^{2}\right)+\frac{l^{m} c}{m(m-2)}\left(1-\frac{1}{r^{m-2}}\right) & \text { if } m \geq 3 .
\end{array}\right.
$$

The value of $c$ is now obtained by the requirement $u(l)=1$,

$$
c= \begin{cases}\left(\frac{1-l^{2}}{4}+\frac{l^{2}}{2} \ln (l)\right)^{-1} & \text { if } m=2, \\ \left(\frac{1}{2 m}+\frac{l^{m}}{m(m-2)}-\frac{l^{2}}{2(m-2)}\right)^{-1} & \text { if } m \geq 3 .\end{cases}
$$

One verifies in a straightforward way that the resulting function $u \equiv u(\cdot ; l)$ is decreasing for $l \leq r \leq 1$, that $\theta(0)=2 /(m+2)$, and that $l \mapsto c \equiv c(l)$ is a continuous, strictly increasing function of $0 \leq l<1$. We claim that $l \mapsto \theta(l)$ is also strictly increasing. To verify that this is indeed the case, one could explicitly compute $\theta$ in terms of $l$, but the formula is rather complicated. Instead we prove the claim by using the maximum principle. By contradiction, suppose there exist $0 \leq l_{2}<l_{1}<1$ with $\theta_{2}:=\theta\left(l_{2}\right)>\theta_{1}:=\theta\left(l_{1}\right)$. By the considerations above, $c_{2}:=c\left(l_{2}\right)<c_{1}:=c\left(l_{1}\right)$. Hence $-\Delta\left(u_{1}-u_{2}\right)=c_{1}-c_{2}>0$ on $B_{1} \backslash \bar{B}_{l_{1}}$ where $u_{j}:=u\left(\cdot, l_{j}\right)$ for $j=1$, 2. Since $\theta_{1}<\theta_{2}$, there exist $l_{1}<r_{1}<r_{2}<1$ so that $\left(u_{1}-u_{2}\right)(r)<0$ for any $r_{1}<r<r_{2}$, contradicting the maximum principle.

From the formula (97) of $u(\cdot ; l)$ one infers that $\theta(l)$ is a continuous function of $l$ and that $\lim _{l \uparrow 1} \theta(l)=1$. Hence for any $0 \leq l<1, u(\cdot ; l)$ coincides with $u_{\theta}$ where $\theta \equiv \theta(l)=f_{B_{1}} u(\cdot ; l)$. Altogether we have shown that $\mathfrak{b}$ is a continuous, increasing bijection.

Define $g:[0,1) \rightarrow \mathbb{R}$ by

$$
g(l):=f(\theta(l))=(1-\theta(l)) \int_{B_{1}}\left|\nabla u_{\theta(l)}\right|^{2}, \quad \theta(l):=\mathfrak{b}^{-1}(l) .
$$

In view of Eq. 76 it then suffices to show that $g$ is increasing on $[0,1)$.

We first consider the case $m=2$. Integrating by parts, one obtains from Eq. 96

$$
\int_{B_{1}}\left|\nabla u_{\theta(l)}\right|^{2}=c(l) 2 \pi \int_{l}^{1} u_{\theta(l)}(r) r d r,
$$

and

$$
\theta(l)=l^{2}+2 \int_{l}^{1} u_{\theta(l)}(r) r d r
$$

Using Eqs. 97 and 98, one infers from Eq. 99 that

$$
g(l)=2 \pi \frac{\left(\frac{1}{16}-\frac{l^{2}}{4}+\frac{3 l^{4}}{16}-\frac{l^{4} \ln (l)}{4}\right)\left(\frac{1}{8}-\frac{l^{4}}{8}+\frac{l^{2} \ln (l)}{2}\right)}{\left(\frac{1}{4}-\frac{l^{2}}{4}+\frac{l^{2}}{2} \ln (l)\right)^{3}},
$$

and a straightforward computation yields

$$
g^{\prime}(l)=\pi \frac{l\left(l^{2}-1\right)\left(-l^{2}+l^{2} \ln (l)+\ln (l)+1\right)\left(-5 l^{4}+4 l^{4} \ln (l)+4 l^{2}+8 l^{2} \ln (l)+1\right)}{\left(1-l^{2}+2 l^{2} \ln (l)\right)^{4}} .
$$

By inspection one verifies that $g^{\prime}(l)>0$ on $(0,1)$. 
Without any additional effort we may consider the general case $m \geq 3$, and follow the line of arguments above. Integrating by parts, one has

$$
\int_{B_{1}}\left|\nabla u_{\theta(l)}\right|^{2}=c(l) m \omega_{m} \int_{l}^{1} u_{\theta(l)}(r) r^{m-1} d r
$$

and one computes that

$$
\theta(l)=l^{m}+m \int_{l}^{1} u_{\theta(l)}(r) r^{m-1} d r .
$$

Using formula (97) for $m \geq 3$, one infers

$$
\int_{l}^{1} u_{\theta(l)}(r) r^{m-1} d r=c(l)\left(\frac{l^{m}\left(l^{2}-1\right)}{2 m(m-2)}+\frac{l^{m+2}-1}{2 m(m+2)}+\frac{l^{m}\left(1-l^{m}\right)}{m^{2}(m-2)}+\frac{1-l^{m}}{2 m^{2}}\right)
$$

so that by Eq. 99

$$
g(l)=c(l) m \omega_{m}\left(1-l^{m}-m \int_{l}^{1} u_{\theta(l)}(r) r^{m-1} d r\right) \int_{l}^{1} u_{\theta(l)}(r) r^{m-1} d r .
$$

In the case $m=3$, one gets in this way

$$
g(l)=\frac{24 \pi}{25} \frac{\left(5\left(1-l^{3}\right)+10 l^{3}\left(1-l^{3}\right)-15 l^{3}\left(1-l^{2}\right)-3\left(1-l^{5}\right)\right)\left(1-l^{5}+5\left(l^{3}-l^{2}\right)\right)}{\left(2 l^{3}+1-3 l^{2}\right)^{3}},
$$

and a lengthy computation leads to the formula

$$
g^{\prime}(l)=\frac{24 \pi}{25} \frac{2 l\left(20 l^{4}+67 l^{3}+84 l^{2}+46 l+8\right)}{(2 l+1)^{4}} .
$$

Clearly, $g^{\prime}(l)>0$ on $(0,1)$ for $m=3 .^{1}$

(d) In this last part we prove that equality in Eq. 9 holds if and only if $\Omega$ is a ball and $u$ is a multiple of the torsion function for that ball. Clearly, if $\Omega$ is a ball and $u$ is a multiple of the torsion function for that ball, then Eq. 9 holds (see Eq. 76). Conversely, assume that equality holds in Eq. 9. We re-scale the measure of $\Omega$ and $L^{\infty}$-norm of $u$ as in the proof of Theorem 3(i). Equality in Eq. 9 implies that $u$ has the same Dirichlet integral as its Schwarz rearrangement $u^{*}$,

$$
\int_{\Omega}|\nabla u|^{2}=\int_{B_{1}}\left|\nabla u^{*}\right|^{2},
$$

and its Schwarz rearrangement is the solution of the obstacle problem on the ball $B_{1}-$ see Eq. 96. In view of the strict monotonicity of $f$ on $\left[\frac{2}{m+2}, \frac{2}{3}\right.$ ) (see part (a)) and the (strict) inequalities obtained above $\frac{2}{3}$ (see parts (a)-(c)), this implies that $\theta=\frac{2}{m+2}$, which corresponds to $l=0$ and to $c=2 m$ (see Eqs. 76, 98). It means that $u^{*}$ is a multiple of the torsion function on $B_{1}$ (see Eq. 96).

In order to justify that $u$ has to be equal to $u^{*}$, recall that Eq. 101 holds and that $u^{*}$, being a multiple of the torsion function on $B_{1}$, has a critical set of zero measure. Equality

\footnotetext{
${ }^{1}$ For general $m \geq 4$, the formula for $g^{\prime}$ can be computed to be a quotient of two polynomials with degrees depending on $m$. We believe that $g^{\prime}(l)$ is strictly positive for every $l$ on $(0,1)$, but a direct proof, covering all dimensions $m \geq 5$, based on the formula of $g^{\prime}$ seems out of reach. For $m=4$ the quotient of the polynomials simplifies, and gives $g(l)=\omega_{4}\left(\frac{16}{3} l^{2}+\frac{16}{9}\right)$. We see that for $m=4, g(1)=\frac{64 \omega_{4}}{9}$ agrees with the value $f(1)$ given in Remark 1 . We also have that $g(0)=\frac{16 \omega_{4}}{3}$ agrees with the value $f(1 / 3)$ from Theorem 3(i). Indeed for $m=4$ and $\theta=\frac{1}{3}$ we have equality in Eq. 9. Note that for $m=4, g(l)$ is increasing on $(0,1)$.
} 
between $u$ and $u^{*}$, up to a translation, comes from the classical result of Brothers and Ziemer [11, Theorem 1.1].

Proof of Theorem 3(ii). The key ingredient into the proof is inequality (9). First note that since for any $t>0, \frac{1}{t^{2}} v_{\Omega}(t x)$ is the torsion function of $\frac{1}{t} \Omega$. Choosing $t=\left(|\Omega| / \omega_{m}\right)^{1 / m}$, one infers that it suffices to prove estimate (10) in the case $|\Omega|=\omega_{m}$.

We apply (i) to

$$
u(x)=\frac{v_{\Omega}(x)}{\left\|v_{\Omega}\right\|_{\infty}}, \quad x \in \Omega .
$$

Observe that $f_{\Omega} u=\Phi(\Omega)$. First we consider the case where $f_{\Omega} u \geq \frac{2}{m+2}$. Then by Eq. 72

$$
\frac{1}{\left\|v_{\Omega}\right\|_{\infty}^{2}}(1-\Phi(\Omega)) \int_{\Omega}\left|\nabla v_{\Omega}\right|^{2} \geq \frac{4 m^{2}}{(m+2)^{2}} \omega_{m} .
$$

Since $-\Delta v_{\Omega}=1$,

$$
\frac{1}{\omega_{m}\left\|v_{\Omega}\right\|_{\infty}} \int_{\Omega}\left|\nabla v_{\Omega}\right|^{2}=\frac{1}{\omega_{m}\left\|v_{\Omega}\right\|_{\infty}} \int_{\Omega} v_{\Omega}=\Phi(\Omega) .
$$

Since $\Phi(\Omega) \leq 1$, we find that

$$
\begin{aligned}
\left\|v_{\Omega}\right\|_{\infty} & \leq \frac{(m+2)^{2}}{4 m^{2}} \Phi(\Omega)(1-\Phi(\Omega)) \\
& \leq \frac{(m+2)^{2}}{4 m^{2}}(1-\Phi(\Omega)),
\end{aligned}
$$

which gives Eq. 10. Next consider the case $f_{\Omega} u \leq \frac{2}{m+2}$. Since by the de Saint-Venant's principle $\left\|v_{\Omega}\right\|_{\infty} \leq\left\|v_{B_{1}}\right\|_{\infty}$ and since $\left\|v_{B_{1}}\right\|_{\infty}=1 / 2 m$ we find that

$$
\begin{aligned}
1-\Phi(\Omega) & \geq \frac{m}{m+2}=\frac{2 m^{2}}{m+2} \cdot \frac{1}{2 m} \\
& \geq \frac{2 m^{2}}{m+2}\left\|v_{\Omega}\right\|_{\infty} .
\end{aligned}
$$

Note that $\frac{2 m^{2}}{m+2} \geq \frac{4 m^{2}}{(m+2)^{2}}$ and hence the estimate (10) also holds in this case.

Inequality (11) follows from Eqs. 1 and 10.

Proof of Theorem 3(iii). The key ingredient in the proof is inequality (9). Since $\Omega$ is connected, $\lambda_{1}(\Omega)$ has multiplicity 1 and hence both, $\varphi_{1, \Omega}$ and $E(\Omega)$, are well defined. First note that since for any $t>0, t^{m / 2} \varphi_{1, \Omega}(t x)$ is the positive $L^{2}$-normalised Dirichlet eigenfunction of $\frac{1}{t} \Omega$, choosing $t=\left(|\Omega| / \omega_{m}\right)^{1 / m}$, one infers that it suffices to prove estimate (12) in the case $|\Omega|=\omega_{m}$. By (26) in [4], one has

$$
\left\|\varphi_{1, \Omega}\right\|_{\infty} \leq\left(\frac{e}{2 \pi m}\right)^{m / 4} \lambda_{1}(\Omega)^{m / 4} .
$$

Hence $\varphi_{1, \Omega} \in L^{\infty}(\Omega)$. We apply (9) to

$$
u(x)=\frac{\varphi_{1, \Omega}(x)}{\left\|\varphi_{1, \Omega}\right\|_{\infty}}, \quad x \in \Omega .
$$


First we consider the case where $f_{\Omega} u \geq \frac{2}{m+2}$. Then by Eq. 72

$$
\left(1-f_{\Omega} u\right) \int_{\Omega}|\nabla u|^{2} \geq \frac{4 m^{2}}{(m+2)^{2}} \omega_{m}
$$

Since $\int_{\Omega}|\nabla u|^{2}=\lambda_{1}(\Omega) \int_{\Omega} u^{2} \leq \lambda_{1}(\Omega) \omega_{m}$, and $f_{\Omega} u=E(\Omega)$ we obtain,

$$
\lambda_{1}(\Omega) \geq \frac{4 m^{2}}{(m+2)^{2}}(1-E(\Omega))^{-1} .
$$

Now let us consider the case where $f_{\Omega} u \leq \frac{2}{m+2}$. Then $1-E(\Omega) \geq \frac{m}{m+2}$, and hence by Faber-Krahn,

$$
\lambda_{1}(\Omega) \geq \lambda_{1}\left(B_{1}\right) \frac{m}{m+2}(1-E(\Omega))^{-1} .
$$

Combining (102) and (103) gives

$$
\lambda_{1}(\Omega) \geq \min \left\{\frac{4 m^{2}}{(m+2)^{2}}, \frac{m}{m+2} \lambda_{1}\left(B_{1}\right)\right\}(1-E(\Omega))^{-1} .
$$

To finish the proof we recall that

$$
\lambda_{1}\left(B_{1}\right)=j_{(m-2) / 2}^{2} .
$$

By the results of [20], we have that

$$
j_{(m-2) / 2}^{2} \geq \frac{m(m+8)}{4} .
$$

Hence by Eqs. 104, 105, 106,

$$
\lambda_{1}(\Omega) \geq \frac{4 m^{2}}{(m+2)^{2}}(1-E(\Omega))^{-1},
$$

which is inequality (12) in the case $|\Omega|=\omega_{m}$.

Remark 2 By an elementary computation, using the expression for $g$ in terms of $l$ from Eq. 100, one can show that $\lim _{\theta \uparrow 1} f(\theta)=\frac{4}{9} m^{2} \omega_{m}, m \geq 2$.

Below we show that $\lambda_{1}(\Omega)$ cannot be bounded from above in terms of $(1-$ $E(\Omega))^{-1}|\Omega|^{-2 / m}$ nor of $(1-\Phi(\Omega))^{-1}|\Omega|^{-2 / m}$.

Remark 3 We have

$$
\sup \left\{\lambda_{1}(\Omega)(1-E(\Omega))|\Omega|^{2 / m}: \Omega \text { open, convex, } 0<|\Omega|<\infty\right\}=\infty,
$$

and

$$
\sup \left\{\lambda_{1}(\Omega)(1-\Phi(\Omega))|\Omega|^{2 / m}: \Omega \text { open, convex, } 0<|\Omega|<\infty\right\}=\infty .
$$

Proof To prove (107) we let $\Omega_{n}=(0,1)^{m-1} \times(0, n)$. Then $\lambda_{1}\left(\Omega_{n}\right) \geq(m-1) \pi^{2}$. A straightforward calculation shows that for an interval of length $L, L>0, E((0, L))=\frac{2}{\pi}$. By separation of variables $E\left(\Omega_{n}\right)=\frac{2^{m}}{\pi^{m}}$. We conclude that the supremum in Eq. 107 is bounded from below by $(m-1)\left(1-\frac{2^{m}}{\pi^{m}}\right) \pi^{2} n^{2 / m}$. Letting $n \rightarrow \infty$ concludes the proof.

To prove (108) we use [15, Theorem 1.1 (i)] for $p=q=2$ to see that $\Phi\left(\Omega_{n}\right) \leq \frac{2}{3}$. We conclude that the supremum in Eq. 108 is bounded from below by $\frac{1}{3}(m-1) \pi^{2} n^{2 / m}$. Letting $n \rightarrow \infty$ concludes the proof. 


\section{Proof of Theorem 4}

We start with the following observation.

Lemma 7 Let $\left(\Omega_{n}\right)$ be a sequence of open sets in $\mathbb{R}^{m}$ with $0<\left|\Omega_{n}\right|<\infty, n \in \mathbb{N}$, $1 \leq p<\infty$, and let $f_{n} \in L^{p}\left(\Omega_{n}\right), n \in \mathbb{N}$ be a sequence of non-negative functions with $0<\left\|f_{n}\right\|_{\infty}<\infty$. If $\left(f_{n}\right)$ either localises in $L^{p}$ or $\kappa$-localises in $L^{p}$ then $\left(f_{n}\right)$ has vanishing mean to max ratio.

Proof Let $\varepsilon \in(0,1)$ be arbitrary. By hypothesis there exists a sequence $\left(A_{n}\right)$ satisfying (14). Then for all $n$ sufficiently large $\left|A_{n}\right| /\left|\Omega_{n}\right|<\varepsilon$, and $\left\|f_{n}\right\|_{p}^{p} \leq \kappa^{-1}(1-\varepsilon)^{-1} \int_{A_{n}} f_{n}^{p}$. Then for all such $n$,

$$
\left\|f_{n}\right\|_{p}^{p} \leq(1-\varepsilon)^{-1} \int_{A_{n}} f_{n}^{p} \leq(1-\varepsilon)^{-1}\left\|f_{n}\right\|_{\infty}^{p}\left|A_{n}\right| \leq \frac{\varepsilon \kappa^{-1}}{1-\varepsilon}\left\|f_{n}\right\|_{\infty}^{p}\left|\Omega_{n}\right| .
$$

By Hölder's inequality,

$$
\left(\int_{\Omega_{n}} f_{n}\right)^{p} \leq\left\|f_{n}\right\|_{p}^{p}\left|\Omega_{n}\right|^{p-1} .
$$

By Eqs. 109 and 110 we have for all $n$ sufficiently large,

$$
\frac{\left\|f_{n}\right\|_{1}}{\left|\Omega_{n}\right|\left\|f_{n}\right\|_{\infty}} \leq\left(\frac{\varepsilon \kappa^{-1}}{1-\varepsilon}\right)^{1 / p}
$$

Since $\varepsilon \in(0,1)$ was arbitrary, $\left(f_{n}\right)$ has vanishing mean to max ratio.

Proof of Theorem 4 We obtain by Eqs. 39 and 51

$$
v_{\Omega, V}(x)=\sum_{j=1}^{\infty} \lambda_{j}(\Omega, V)^{-1}\left(\int_{\Omega} \varphi_{j, \Omega, V}\right) \varphi_{j, \Omega, V}(x) .
$$

Integrating with respect to $x$ over $\Omega$ gives

$$
\begin{aligned}
\int_{\Omega} v_{\Omega, V} & =\sum_{j=1}^{\infty} \lambda_{j}(\Omega, V)^{-1}\left(\int_{\Omega} \varphi_{j, \Omega, V}\right)^{2} \\
& \geq \lambda_{1}(\Omega, V)^{-1}\left(\int_{\Omega} \varphi_{1, \Omega, V}\right)^{2}
\end{aligned}
$$

Multiplying both sides with $\lambda_{1}(\Omega, V)$, and using the definition of $\mathfrak{d}_{m}$ in Eq. 42 gives

$$
\mathfrak{d}_{m} \frac{\left\|v_{\Omega, V}\right\|_{1}}{\left\|v_{\Omega, V}\right\|_{\infty}} \geq\left(\int_{\Omega} \varphi_{1, \Omega, V}\right)^{2} .
$$

This implies that

$$
\mathfrak{d}_{m} \Phi(\Omega, V) \geq \frac{1}{|\Omega|}\left(\int_{\Omega} \varphi_{1, \Omega, V}\right)^{2} .
$$

Suppose $\left(v_{\Omega_{n}, V_{n}}\right)$ either localises or $\kappa$ - localises in $L^{1}$. By Lemma 7 for $p=1$, $\lim _{n \rightarrow \infty} \Phi\left(\Omega_{n}, V_{n}\right)=0$. By Eq. 111, $\lim _{n \rightarrow \infty} \frac{1}{\left|\Omega_{n}\right|}\left(\int_{\Omega_{n}} \varphi_{1, \Omega_{n}, V_{n}}\right)^{2}=0$. This implies local-

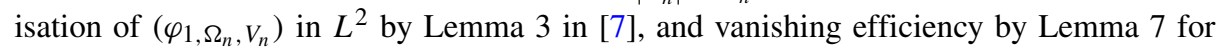
$p=2$. 
Theorem 4 implies that if $\left(\Omega_{n}, V_{n}\right)$ satisfies the $\eta$ condition of Theorem 1(ii), and if either $\left(v_{\Omega_{n}}\right)$ or $\left(v_{\Omega_{n}, V_{n}}\right)$ have non-vanishing efficiencies then both $\left(v_{\Omega_{n}}\right)$ and $\left(v_{\Omega_{n}, V_{n}}\right)$ are not localising.

Acknowledgements MvdB and TK acknowledge support by the Leverhulme Trust through Emeritus Fellowship EM-2018-011-9, and the Swiss National Science Foundation respectively. DB was supported by the LabEx PERSYVAL-Lab GeoSpec (ANR-11-LABX-0025-01) and ANR SHAPO (ANR-18-CE40-0013).

Open Access This article is licensed under a Creative Commons Attribution 4.0 International License, which permits use, sharing, adaptation, distribution and reproduction in any medium or format, as long as you give appropriate credit to the original author(s) and the source, provide a link to the Creative Commons licence, and indicate if changes were made. The images or other third party material in this article are included in the article's Creative Commons licence, unless indicated otherwise in a credit line to the material. If material is not included in the article's Creative Commons licence and your intended use is not permitted by statutory regulation or exceeds the permitted use, you will need to obtain permission directly from the copyright holder. To view a copy of this licence, visit http://creativecommons.org/licenses/by/4.0/.

\section{References}

1. Arnold, D.N., David, G., Filoche, M., Jerison, D., Mayboroda, S.: Computing spectra without solving eigenvalue problems. SIAM J. Sci. Comput. 41, B69-B92 (2019)

2. Arnold, D.N., David, G., Filoche, M., Jerison, D., Mayboroda, S.: Localization of eigenfunctions via an effective potential. Commun. Partial. Differ. Equ. 44, 1186-1216 (2019)

3. Beck, T.: The torsion function of convex domains of high eccentricity. Potential Analy. 53, 701-726 (2020)

4. van den Berg, M.: Estimates for the torsion function and Sobolev constants. Potential Analy. 36, 607-616 (2012)

5. van den Berg, M.: Spectral bounds for the torsion function. Integr. Equ. Oper. Theory 88, 387-400 (2017)

6. van den Berg, M., Carroll, T.: Hardy inequality and $L^{p}$ estimates for the torsion function. Bull. Lond. Math. Soc. 41, 980-986 (2009)

7. van den Berg, M., Della Pietra, F., di Blasio, G., Gavitone, N.: Efficiency and localisation for the first Dirichlet eigenfunction. J. Spectr. Theory, to appear. arXiv:1905.06591

8. van den Berg, M., Ferone, V., Nitsch, C., Trombetti, C.: On Pólya's inequality for torsional rigidity and first Dirichlet eigenvalue. Integr. Equ. Oper. Theory 86, 579-600 (2016)

9. van den Berg, M., Kappeler, T.: Localization for the torsion function and the strong Hardy inequality. Mathematika 67, 514-531 (2021)

10. van den Berg, M., Srisatkunarajah, S.: Heat flow and Brownian motion for a region in $\mathbb{R}^{2}$ with a polygonal boundary. Probab. Theory Relat. Fields 86, 41-52 (1990)

11. Brothers, J.E., Ziemer, W.P.: Minimal rearrangements of Sobolev functions. J. für die Reine und Angew. Math. 384, 153-179 (1988)

12. Bucur, D., Buttazzo, G.: Variational methods in shape optimization problems. Progress in Nonlinear Differential Equations and their Applications, vol. 65. Birkhäuser Boston, Inc, Boston, MA (2005)

13. Cioranescu, D., Murat, F.: A strange term coming from nowhere. Topics in the mathematical modelling of composite materials, vol. 31, pp. 45-93. Birkhäuser, Boston (1997)

14. Dal Maso, G., Mosco, U.: Wiener's criterion and $\Gamma$-convergence. Appl. Math. Optim. 15, 15-63 (1987)

15. Della Pietra, F., Gavitone, N., Guarino Lo Bianco, S.: On functionals involving the torsional rigidity related to some classes of nonlinear operators. J. Differ. Equ. 265, 6424-6442 (2018)

16. Eisen, G.: The obstacle problem with a volume constraint. Manuscripta Mathematica 43, 73-83 (1983)

17. Giorgi, T., Smits, R.G.: Principal eigenvalue estimates via the supremum of torsion. Indiana Univ. Math. J. 59, 987-1011 (2010)

18. Grebenkov, D.S., Nguyen, B.-T.: Geometrical structure of Laplacian eigenfunctions. SIAM Rev. 55, 601-667 (2013)

19. Henrot, A., Lucardesi, I., Philippin, G.: On two functionals involving the maximum of the torsion function. ESAIM Control Optim. Calc. Var. 24, 1585-1604 (2018)

20. Lorch, L.: Some inequalities for the first positive zeros of Bessel functions. SIAM J. Math. Anal. 24, 814-823 (1993) 
21. Payne, L.E., Stakgold, I.: On the mean value of the fundamental mode in the fixed membrane problem. Collection of articles dedicated to Alexander Weinstein on the occasion of his 75th birthday. Applicable Anal. 3, 295-306 (1973)

22. Simon, B.: Functional integration and quantum physics Pure and Applied Mathematics, vol. 86. Academic Press, Inc. [Harcourt Brace Jovanovich, Publishers], New York-London (1979)

23. Sperb, R.: Maximum principles and applications. Academic Press, Inc. [Harcourt Brace Jovanovich Publishers], New York-London (1981)

24. Vogt, H.: $L_{\infty}$ - estimates for the torsion function and $L_{\infty^{-}}$growth of semigroups satisfying Gaussian bounds. Potential Anal. 51, 37-47 (2019)

Publisher's Note Springer Nature remains neutral with regard to jurisdictional claims in published maps and institutional affiliations. 